\title{
Correlation Analysis of High Resolution Particle Image Velocimetry Data of Screeching Jets
}

\author{
D.J. Tan* and D. Honnery ${ }^{\dagger}$, \\ Monash University, Clayton, Victoria, 3800, Australia \\ A. Kalyan, V. Gryazev ${ }^{\S}$, and S.A. Karabasov ${ }^{\mathbb{I}}$, \\ Queen Mary University of London, Mile End Road, London E1 4NS, United Kingdom \\ D. Edgington-Mitchell \\ Monash University, Clayton, Victoria, 3800, Australia
}

The acoustic analogy provides a general framework for predicting broadband jet noise. The accuracy of the noise predictions are strongly dependent on the second- and fourth-order integral time and length scales of the turbulence quantities in the jet. Two low-order models for the second- and fourth-order integral length scales are examined. The low-order models are defined by locally isotropic scales estimated from 2D particle image velocimetry measurements. These measurements are of screeching underexpanded unheated round jets issuing from a purely converging nozzle at conditions, which corresponds to ideally expanded Mach numbers of 1.45 and 1.59. The jets are dominated by the helical $C$ instability screech mode, which is associated with large-scale coherent periodic fluctuations. These fluctuations are filtered using a proper orthogonal decomposition method to assess low-order models that approximate the length scales associated with the broadband noise mechanisms. The length scale model parameters are shown to be insensitive for the two Mach numbers considered. The root-mean-square error associated with the low-order models indicates that either is sufficient for approximating the integral length scales required to model equivalent sources of broadband jet noise. 


\section{Nomenclature}

$\alpha$ Mixed-scale model parameter

$\boldsymbol{\eta}$ Displacement vector, $(\xi, \eta, \zeta)$

$\psi$ Eigenfunctions (POD modes)

$\boldsymbol{e}_{i}$ Eigenvectors

$\boldsymbol{R}$ Auto-covariance matrix

$\boldsymbol{x}$ Observer location

$y$ Source location, $(x, y, z)$

$\Delta\left(c_{L}\right)$ Relative discrepancy

$\hat{\tau}$ Functional time scale

$\hat{L}$ Functional length scale

$\kappa$ Wave number

$\Lambda$ Upper limit of integral length scale

$\lambda_{i}$ Eigenvalues

q State vector

$\mathcal{L}$ Linear operator

$\overline{V_{i}}$ Mean velocity

$\bar{v}_{i}$ Mean velocity

$\psi$ Stream function

Re Reynolds number

$\tau$ Time separation

$\tau_{\mathrm{s}}$ Integral time scale

*Ph.D. Student, Laboratory for Turbulence Research in Aerospace and Combustion, Department of Mechanical and Aerospace Engineering, dominic.tan@monash.edu, and AIAA Member

${ }^{\dagger}$ Professor, Laboratory for Turbulence in Aerospace and Combustion, Department of Mechanical and Aerospace Engineering, damon.honnery@monash.edu, and AIAA Member

‡Ph.D Student, School of Engineering and Material Sciences, a.kalyan@qmul.ac.uk, and AIAA member

\$Ph.D Student, School of Engineering and Material Sciences, v.gryazev@qmul.ac.uk, and AIAA member

${ }^{I}$ Reader in Computational Modelling, School of Engineering and Material Sciences, s.karabasov@qmul.ac.uk, and AIAA Senior Member

"Senior Lecturer, Laboratory for Turbulence Research in Aerospace and Combustion, Department of Mechanical and Aerospace Engineering, daniel.mitchell@monash.edu, and AIAA Member 
$c_{\tau}$ Time scale coefficient

$c_{L}$ Length scale coefficient

$D$ Nozzle diameter

$f$ Non-dimensional time scale function

$k$ turbulence kinetic energy

$L$ Integral length scale

$M$ Mach number

$R^{v}$ Velocity correlation function

$r^{v}$ Velocity correlation coefficients

$s(\boldsymbol{y}, t)$ Equivalent BBSAN source

$t$ Time

$v_{i}$ Velocity fluctuations

Subscripts

0 Stagnation condition

$\infty$ Free-stream condition

c Convective component

$e$ Exit condition

j Ideally expanded condition

Superscripts

(1) Streamwise direction

(2) Transverse direction

' Random fluctuating component

" Random fluctuating stress

c Coherent velocity component

$v$ Velocity component 


\section{Introduction}

Supersonic jets at take-off radiate significant noise, which consists of shock-associated and turbulence mixing components. The shock-associated components are radiated in the sideline and upstream directions, where the latter is associated with the mid- to high frequency noise. The turbulence mixing noise is primarily radiated in the aft direction of the jet. The mixing noise is generated from a broad range of turbulent eddies, which results in non-compact sources due to the large range of space and time scales. The large-scale turbulence also interacts with a quasi-periodic shock cell structure that is present at off-design conditions. The turbulence/shock interactions contribute to the generation of shock-associated noise, which includes both discrete tones (screech) and broadband components (BBSAN). The screech tones, which have a strong impact on jet dynamics, are more prevalent in laboratory-scale supersonic jets. The screech mechanism is characterised by a self-reinforcing acoustic resonance phenomenon [1] that is associated with high-energy periodic large-scale turbulence fluctuations in the shear layer interacting with the tips of the shock cells. Weak interactions between random large-scale turbulence fluctuations and the shock cells results in the generation of BBSAN [2]. Predictive models for BBSAN scale with the second-order velocity correlations $[3,4]$, whereas the mixing noise scales by the auto-covariance of generalised turbulent fluctuating stresses [5], which reduce to the fourth-order velocity auto-correlation functions for unheated jets.

The mixing noise and BBSAN radiated by a supersonic screeching jet may be described through a manipulation of the Navier-Stokes equations. Numerical methods that directly solve the Navier-Stokes equations are computationally expensive due to large differences between acoustic and local turbulence scales. Hybrid approaches reduce the computational costs by decoupling the acoustic propagation from its generation by the local turbulent flow-field. A precursor to hybrid approaches is Lighthill's acoustic analogy [6], which is formulated through a rearrangement of the Navier-Stokes equations to provide a general framework for the development of jet noise prediction schemes.

The equivalent sources can be estimated from large eddy simulations (LES) and de- 
tached eddy simulations (DES), with the noise propagation handled using integral methods involving Kirchhoff [7,8] or Ffowcs Williams-Hawking [9] surfaces. Non-linear propagation effects can be accounted for by solving a simplified set of equations that govern the acoustic field $[10,11]$. The computational cost associated with LES and DES is still significant; steady Reynolds-Averaged Navier-Stokes (RANS) solvers with a $k-\epsilon$ [12] or $k-\omega[3,4]$ turbulence closure model may be favoured for rapid acoustic predictions [3,12-14].

Reliable noise predictions from RANS solutions depend strongly on a consistent definition of equivalent acoustic sources that sufficiently captures the dominant noise mechanisms [15]. The noise in the context of an acoustic analogy scales with the spatio-temporal velocity correlations, which define the equivalent acoustic sources. These sources are approximated by a statistical model that is a function of the mean velocity, turbulence kinetic energy, and dissipation rate. These properties can be extracted from RANS solutions with a two-equation turbulence model. The statistical model separates the spatial and temporal characteristics, which are theoretically given by integral length and time scales, respectively. The integral scales are important for accurate noise predictions [21-24] as they describe the temporal and spatial behaviour of the noise sources. The integral scales, which can be difficult to interpret, are usually modelled based on the local mean turbulence statistics. These models generally enable a representation of the integral scales that can be understood in terms of the underlying physics of the jet turbulence that generates the noise. The simplest low-order integral scale model assumes that the turbulence scales are directly proportional to functional scales defined by ratios of the local turbulence statistics [16].

The proportionality coefficients for the correlation scale models have previously been determined by matching jet noise predictions to far-field acoustic measurements at a single operating condition and observer location [3,4,17]. Karabasov et al. [13] presented coefficients for modelling the fourth-order coefficients by fitting a mixing noise source model to large eddy simulations (LES) and solutions to the Reynolds-Averaged Navier-Stokes (RANS) equations with a two-equation turbulence model. The proportion- 
ality coefficients for the time and length scales were determined by fitting the acoustic source models based on mean turbulence properties from the RANS solutions to the spatio-temporal correlations calculated directly from the LES data. The proportionality coefficients for the fourth-order scales were shown to be approximately independent of the jet operating condition. The idea is now extended to the second-order length scales relevant to BBSAN to examine if there are constant proportionality coefficients that hold for multiple conditions.

This paper provides an assessment of the assumptions made in modelling the secondand fourth-order length scales. The length scales are approximated by two low-order models, which are appropriate for use in noise prediction schemes such as those based on the acoustic analogy. The low-order models are compared to integral length scales, which are both calculated directly from high spatial resolution 2D particle image velocimetry (PIV) measurements. The measurements are of screeching supersonic axisymmetric unheated jets that issue from a purely converging round nozzle such that the velocity is sonic at the exit. Jets corresponding to ideally expanded Mach numbers of 1.45 and 1.59 are considered, where the ideally expanded Mach number corresponds to the value if the jet isentropically expands to the ambient pressure [2]. The velocity fluctuations associated with the broadband noise mechanisms are studied by filtering the high-energy, large-scale periodic fluctuations associated with the screech mechanism a posteriori [18]. The accuracy of the low-order models are then examined through a root-mean-square error (RMSE) analysis.

\section{Acoustic Analogies}

The equivalent sources in an acoustic analogy represent the noise generation by the effective turbulent noise sources as well as the shock effects for imperfectly expanded jets. Acoustic analogies with a propagator differential operator can generally be expressed as

$$
\mathcal{L} p_{\mathrm{a}}(\boldsymbol{y}, t)=s(\boldsymbol{y}, t)
$$


where $\mathcal{L}$ is a linear operator describing the acoustic propagation, $p_{\mathrm{a}}$ is the acoustic pressure, and $s$ is representative of the equivalent sources that generate noise. The linear wave equation is a valid model of jet noise propagation at large distances from the jet, and angles close to $90^{\circ}$ that is of relevance for shock-associated noise. The DNS computation of Freund et al. [10] indicates that linear propagation modelling of shock-associated noise provides a less expensive model for jet noise propagation up to 35 nozzle radii from the jet. This includes angles close to $90^{\circ}$, which is of relevance for shock-associated noise. However, Freund et al. [10] considered a low Reynolds number flow, which limits the range of broadband scales. Furthermore, atmospheric absorption is a function of absolute frequency for air such that full-scale jet noise is subject to a higher degree of non-linear propagation. The dependence of atmospheric absorption on absolute frequency restricts the acoustic similarity between laboratory-scale, numerical, and full-scale jet noise studies. Replicating full-scale non-linear propagation effects in laboratory-scale jet noise studies is geometrically limited such that novel prediction schemes can complement acoustic experiments.

Under the assumption that the far-field acoustic propagation process does not affect the process of sound generation in the jet, the sound source is modelled separately from propagation. This assumption is naturally satisfied for jet mixing noise of perfectly expanded jets where the acoustic component typically is a very small part of the hydrodynamic flow. For shock-associated noise, the near-field acoustic waves emerging from shock/vortex interactions can be strong enough to affect both the turbulence and the shock structure and, thus, the entire jet flow. Nevertheless, despite the underlying complexity of the interior shock/vortex interaction processes, the entire jet flow at off-design conditions can be modelled separately with respect to the far-field sound propagation for the purpose of reduced-order sound predictions, as shown by Morris and Miller [19]. Once the source computation is separated from the far-field noise propagation, this reduces the problem of computing noise at the far-field observer location to one of solving the inhomogeneous linear wave equation. The latter is amenable to analytical integration methods based on 
the Green's function.

The equivalent (nominal) acoustic sources in an acoustic analogy can be expressed in terms of the spatio-temporal velocity correlation function $R^{\mathrm{v}}(\boldsymbol{y}, t, \boldsymbol{\eta}, \tau)$. These correlations can be approximated [3] by assuming that the time and space variables are separable [20] so that

$$
R^{\mathrm{v}}(\boldsymbol{y}, \boldsymbol{\eta}, \tau)=R^{\mathrm{v}}(\boldsymbol{y}, 0,0) e^{-|\tau| / \tau_{s}} e^{-\left(\xi-\bar{v}_{\mathrm{c}} \tau\right)^{2} / L^{(1)^{2}}} e^{-\left(\eta^{2}+\zeta^{2}\right)^{2} / L^{(2)^{2}}}
$$

where $R^{\mathrm{v}}(\boldsymbol{y}, 0,0)$ is the auto-covariance of the velocity fluctuations, $\overline{v_{\mathrm{c}}}$ is the convective velocity, and the temporal and spatial characteristics are described by time scales $\tau_{s}$, and streamwise $\left(L^{(1)}\right)$ and transverse $\left(L^{(2)}\right)$ length scales. The correlation model given by Eq.(2) is a simplified form that is representative of an axially convected turbulence pattern that is random in space and time. The correlation model is an exponential function, so does not capture the negative correlations associated with the screech-related fluctuations, which are evident in the second-order correlations and for large separation distances. The negative correlations are observed as slowly decaying and oscillating functions that are indicative of non-compact source effects associated with large-scale turbulent structures $[25,26]$. Using an estimate for the axial length scale is thus an empirical approximation. The correlation model could be improved by including the negative correlations by a fitting function [13], or by considering multiple length and time scale components [27].

\section{Correlation Scales}

The time and length scales contained in Eq.(2) are theoretically defined by the distribution

of the two-point temporal and spatial velocity correlation coefficients, respectively. The second- and fourth-order spatio-temporal correlation coefficients, respectively, are

$$
r_{i j}^{v}(\boldsymbol{y}, \boldsymbol{\eta}, \tau)=\frac{\left\langle v_{i}^{\prime}(\boldsymbol{y}, t) v_{j}^{\prime}(\boldsymbol{y}+\boldsymbol{\eta}, t+\tau)\right\rangle}{\sqrt{\left\langle v_{i}^{\prime 2}\right\rangle(\boldsymbol{y})\left\langle{v_{j}^{\prime}}^{2}\right\rangle(\boldsymbol{y}+\boldsymbol{\eta})}}
$$


and

$$
r_{i j k l}^{v}(\boldsymbol{y}, \boldsymbol{\eta}, \tau)=\frac{\left\langle v_{i j}^{\prime \prime}(\boldsymbol{y}, t) v_{k l}^{\prime \prime}(\boldsymbol{y}+\boldsymbol{\eta}, t+\tau)\right\rangle}{\sqrt{\left\langle{v_{i j}^{\prime \prime 2}}^{\prime}\right\rangle(\boldsymbol{y})\left\langle{v_{k l}^{\prime \prime 2}}^{\prime 2}\right\rangle(\boldsymbol{y}+\boldsymbol{\eta})}}
$$

where $i, j, k$, and $l$ correspond to the velocity component that is correlated, $\boldsymbol{\eta}=(\xi, \eta, \zeta)$ is the separation distance, $\tau$ is the time delay, and $v^{\prime}$ and $v^{\prime \prime}$ denote fluctuating velocities and stresses, respectively. The fluctuating variables are defined about their mean according to the study of Morris and Zaman [19]. In the same study, the correlation scales along the nozzle lip line were shown to be important for modelling the noise sources. Additional studies that examined the velocity correlations have shown that contributions of the 11,22 , $1111,2222,3333,1212,1313$, and 2323 components dominate in axisymmetric jets [13,28].

The second- and fourth-order integral scales describe the turbulent eddies that extract energy from the mean flow. The integral scales are determined from the shape of the two-point velocity correlation coefficients given by Eq.(3) such that

$$
\tau_{s}(\boldsymbol{y}, \boldsymbol{\eta}=0, \tau)=\int_{0}^{\Lambda} r_{i j}^{v}(\boldsymbol{y}, \boldsymbol{\eta}=0, \tau) \mathrm{d} \tau
$$

and

$$
L_{i j}^{(\phi)}(\boldsymbol{y}, \boldsymbol{\eta}, \tau=0)=\int_{0}^{\Lambda} r_{i j}^{v}(\boldsymbol{y}, \boldsymbol{\eta}, \tau=0) \mathrm{d} \boldsymbol{\eta}
$$

where $\phi$ refers to the streamwise (1) or radial (2) direction, and $\Lambda$ is the upper limit of integration. An appropriate criterion for $\Lambda$ is based on the location of the first zerocrossing [29] $\left(r^{v}(\boldsymbol{x}, \boldsymbol{\eta}=\Lambda)=0\right)$, but can be susceptible to low-correlation noise [18, 30,31]. 
The fourth-order integral time and length scales follow such that

$$
\begin{gathered}
\tau_{i j k l, s}(\boldsymbol{x}, \boldsymbol{\eta}=0, \tau)=\int_{0}^{\Lambda} r_{i j k l}^{v}(\boldsymbol{x}, \boldsymbol{\eta}=0, \tau) \mathrm{d} \tau \\
L_{i j k l}^{(\phi)}(\boldsymbol{x}, \boldsymbol{\eta}, \tau=0)=\int_{0}^{\Lambda} r_{i j k l}^{v}(\boldsymbol{x}, \boldsymbol{\eta}, \tau=0) \mathrm{d} \boldsymbol{\eta}
\end{gathered}
$$

\section{A. Correlation Scale Models}

In the absence of spatially and temporally resolved data, the integral scales may instead be approximated via relationships to functional scales. The functional time and length scales, $\hat{\tau}$ and $\hat{L}$, respectively, are formulated as ratios of the mean turbulence statistics with dimensions of time or length [16]. The time and length scales important for noise source modelling are generally assumed to be directly proportional to the functional scales via fixed coefficients that are valid over a range of jet conditions.

A two-time-scale approach for modelling the source scales was implemented by Kalyan and Karabasov [4] to explicitly take into account the dependence of the BBSAN generation mechanism on the strength of the shock cells. The generation mechanism is described by two random processes with time scales $\hat{\tau}_{1}$ and $\hat{\tau}_{2}$. The time scales $\hat{\tau}_{1}$ and $\hat{\tau}_{2}$ are associated with the random small- and large-scale turbulence, and shock cell effects, respectively. The time scale $\hat{\tau}_{2}$ represents the correlated part of the noise generation mechanism, which is inversely proportional to the shock cell gradient [4] such that

$$
\hat{\tau}_{2} \sim\left|\partial \overline{V_{1}} / \partial x_{1}\right|^{-1}
$$

where $\overline{V_{1}}$ is the mean axial velocity. Low-order models for the random large-scale turbulence fluctuations that are denoted by $\hat{\tau}_{1}$ are considered in the present study. 
The time scale $\hat{\tau}_{s}$ that is dependent on $\hat{\tau}_{1}$ and $\hat{\tau}_{2}$ is modelled according to

$$
\hat{\tau}_{\mathrm{s}}=c_{\tau} \hat{\tau}_{1} \cdot \begin{cases}f\left(\hat{\tau}_{1}, \hat{\tau}_{2}\right), & \text { if } f\left(\hat{\tau}_{1}, \hat{\tau}_{2}\right)>1 \\ 1, & \text { else }\end{cases}
$$

where $f\left(\hat{\tau}_{1}, \hat{\tau}_{2}\right)$ is a non-dimensional power law defined as

$$
f\left(\hat{\tau}_{1}, \hat{\tau}_{2}\right)=\left(\frac{\hat{\tau}_{1}}{\hat{\tau}_{2}}\right)^{\alpha}
$$

In this relation, $\alpha$ controls the relative contributions of the two time scales with values of 0 or 2 for the BBSAN acoustic analogy model of Morris and Miller [3], or the mixed-scale model of Kalyan et al. [4], respectively. For the mixed-scale model, in regions where the shocks are weak, the function tends to 1 so that only contributions associated with $\tau_{1}$ are considered. The functional streamwise length scales are estimated based on a mean square procedure that is applied to the integral length scale such that

$$
\hat{L}^{(1)}=c_{L^{(1)}} \sqrt{k} \hat{\tau}_{s}
$$

where $\hat{\tau}_{s}$ was previously defined to be proportional to ratios of the local turbulence statistics. The transverse length scale is determined based on an anisotropy relationship given by

$$
\hat{L}^{(2)}=c_{L^{(2)}} \hat{L}^{(1)}
$$

The proportionality coefficients $c_{\tau}, c_{L^{(1)}}$, and $c_{L^{(2)}}$ are usually determined by fitting predictions using the statistical source model to far-field measurements in the sideline direction for one condition $[3,4]$. The coefficients primarily control the shape of the acoustic spectrum and its magnitude [3]. The coefficients are typically fixed for a given operating regime, such as over- or underexpanded conditions. For fixed coefficient values, NPR, and total temperature ratio (TTR) scaling can be accounted for using an equivalent source 
that depends on the local density, speed of sound, and streamwise velocity components with a vector Green's function [33]. Consequently, temperature effects are not directly considered in this study as additional data is required to quantify the effects of TTR on the proportionality coefficients.

\section{B. Time Scale Definitions}

There are several methods by which $\hat{\tau}_{1}$ can be defined. Two definitions for $\hat{\tau}_{1}$ that are dependent on the turbulence dissipation and large eddy convection time are now considered.

\section{Turbulence Energy Dissipation Time Scale}

Following Tam and Auriault [17], Morris and Miller [3] considered the random large-scale turbulence scales defined by the turbulence dissipation given by

$$
\hat{\tau}_{1} \sim \frac{k}{\epsilon}
$$

with the length scales defined by Eq. (12) and (13). The proportionality coefficient $\left(c_{\tau}\right)$ used by Morris and Miller [3] was determined by matching predictions to far-field measurements for a single jet condition and observer location. The streamwise length scale coefficient was determined to be 3.25 , which was noted to be much larger than the value used by Tam and Auriault in their model of fine-scale turbulent mixing noise [17]. The difference was ascribed to the fact that BBSAN is controlled by the large-scale structures in the jet shear layer that are coherent over relatively large axial distances.

\section{Eddy Convection Time Scale}

An alternative time scale that is commonly used to model the turbulence considers the large-scale eddies with a characteristic convection velocity. The time scale is assumed to 
scale with the large-eddy turnover time, which is given by

$$
\hat{\tau}_{1} \sim \frac{D_{\mathrm{j}}}{\overline{V_{1}}}
$$

where the mean axial velocity $\overline{V_{1}}$ is used to approximate the convection of the turbulent eddies in the jet shear layers, which are the important locations for jet noise $[19,34]$. The large-eddy turnover time is the typical time scale for an eddy of length scale $L$ to undergo significant distortion [35]. This is also the typical time scale for the transfer of energy from scale $L$ to smaller scales, since this distortion is the mechanism for energy transfer. Notably, Eq.(15) contains the ideally expanded jet diameter rather than the nozzle diameter. However, the former is a well-defined function of the jet flow. That is, it does not depend on the far-field acoustic variable. Therefore, an acoustic model that uses the suggested convection velocity scale for far-field noise predictions would be closed.

\section{LTRAC Jets}

Table 1. Summary of the jet parameters for the PIV experiments

\begin{tabular}{lcc}
\hline \hline & \multicolumn{2}{c}{ PIV } \\
\cline { 2 - 3 }$M_{\mathrm{j}}$ & 1.45 & 1.59 \\
$\mathrm{NPR}$ & 3.4 & 4.2 \\
$D_{\mathrm{j}}(\mathrm{mm})$ & 16.02 & 16.73 \\
$\operatorname{Re}_{\mathrm{j}}\left(\times 10^{5}\right)$ & 8.51 & 10.6 \\
\hline \hline
\end{tabular}

The velocity correlations are calculated using the turbulence data from spatially resolved two-dimensional two-component (2D-2C) PIV measurements. The measurements are of supersonic unheated jets with ideally expanded Mach numbers $M_{\mathrm{j}}$ of 1.45 and 1.59. These jets radiate screech in the helical C instability mode [36], with a higher intensity for $M_{\mathrm{j}}=1.45$ [37] such that greater turbulence amplification is expected. The correlation scales associated with the broadband noise mechanisms are studied by filtering the fluctuations associated with the screech mechanism using a proper orthogonal decompo- 
sition (POD) method [18]. The POD-based method avoids the use of intrusive devices that can modify the overall shock-cell structure [38], but retains any tone-induced amplification effects due to the presence of screech. Results from an investigation by Tan et al. [18] suggest that the integral length scales of the filtered velocity fluctuations normalised by the incompressible momentum thickness are consistent with non-screeching jet studies. Correlation scale coefficients are determined by the ratio of the integral and functional scales along the nozzle lip line, which is the region identified by Morris and Zaman [19] to be important for modelling the noise sources. A summary of the jet conditions considered is provided in Table 1. Additional details on the screech mechanism and internal flow structure of these jets has been presented in previous work [36,37].

\section{A. Supersonic Jet Facility}

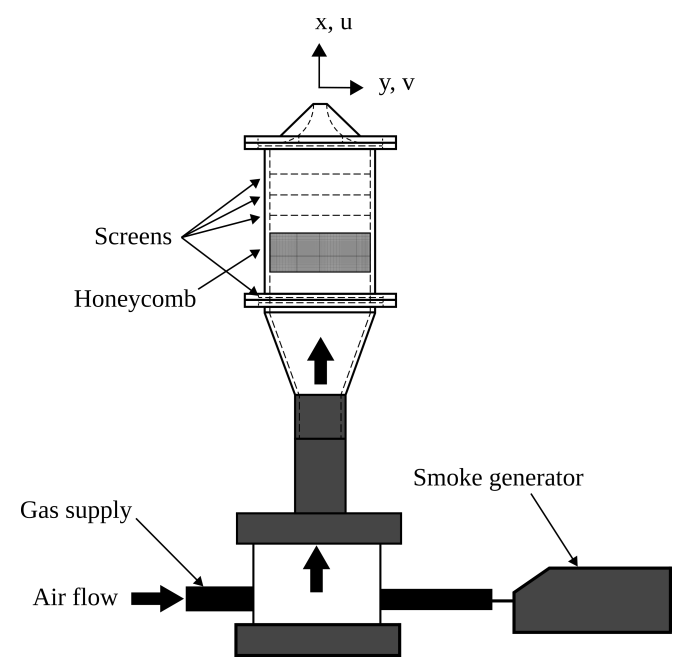

Figure 1. Schematic of the LTRAC Supersonic Jet Facility [36]

PIV jet experiments were conducted in the Laboratory for Turbulence Research in Aerospace and Combustion (LTRAC) Supersonic Jet Facility at Monash Univeristy. A schematic of the facility is shown in Fig. 1. The facility was designed to maximise optical access for various unheated jet configurations. The facility supplies compressed air to the plenum chamber at approximately $288 \mathrm{~K}$. The compressed air is connected directly to the mixing chamber where the free stream and jet are uniformly seeded with oil particles 
from a Vicount 1300 smoke generator. The mixing chamber is connected to the plenum chamber, which contains a honeycomb section and wire mesh screens to condition the flow. The nozzle pressure ratio $\left(N P R=P_{0} / P_{\infty}\right)$ is maintained within $1 \%$ via a Fairchild 100 High Flow pressure regulator. The stagnation pressure in the plenum is measured using an RS-461 pressure transducer with an uncertainty of $\pm 0.25 \%$ over a range of $0-10$ bar. Compressed air issues from a $15 \mathrm{~mm}$ diameter axisymmetric nozzle with a sharp-edged lip with a thickness of $5 \mathrm{~mm}$. The nozzle has an inlet to exit area ratio of 93.44 with a short purely converging section so that the flow is sonic at the exit with a velocity of $V_{\mathrm{e}}=310 \mathrm{~m} / \mathrm{s}$. The converging section consists of a contoured wall with a radius of curvature of $67.15 \mathrm{~mm}$ and a short parallel section at the exit. A complete description of the facility and the PIV system can be found in previous work $[36,37,39]$. Complications specific to the application of PIV to supersonic flows in this facility have been thoroughly investigated [40].

\section{B. PIV Measurements}

Table 2. Non-dimensional PIV parameters for a multigrid cross-correlation algorithm [41]

\begin{tabular}{lcc}
\hline \hline Parameter & Value & $\begin{array}{c}\text { Non-dimensional } \\
\text { value }\end{array}$ \\
\hline Total number of vectors & $182 \times 1000$ & - \\
$\mathrm{IW}_{0}$ & 64 pixels & $0.10 \mathrm{D}$ \\
$\mathrm{IW}_{1}$ & 16 pixels & $0.025 \mathrm{D}$ \\
Grid spacing & 8 pixels & $0.013 \mathrm{D}$ \\
Depth of field & $2.4 \mathrm{~mm}$ & $0.17 \mathrm{D}$ \\
Light sheet thickness & $1.5 \mathrm{~mm}$ & $0.1 \mathrm{D}$ \\
$\Delta t$ & $480 \mathrm{~ns}$ & - \\
Field of view & $150 \mathrm{~mm} \times$ & $10 \mathrm{D} \times 2.2 \mathrm{D}$ \\
Maximum resolvable velocity & $35 \mathrm{~mm}$ & $230 \mathrm{~m} / \mathrm{s}$ \\
Minimum resolvable velocity & $6 \mathrm{~m} / \mathrm{s}$ & $0.02 V_{\mathrm{e}}$ \\
\hline \hline
\end{tabular}

The experiments yielded 8500 statistically independent velocity fields for each jet 
condition. The optical arrangement had two orthogonally mounted PCO 4000 cameras, each with a full array size of $4008 \times 2672$ pixels. Each camera was fitted with $105 \mathrm{~mm}$ Micro Nikkor Nikon lenses set to an aperture of $f / 5.6$. The resulting images have an optical resolution of $23 \mu \mathrm{m} /$ pixel. A $1.5 \mathrm{~mm}$ plane was illuminated by a NewWave Solo PIV Nd:YAG laser at $532 \mathrm{~nm}$ wavelength, which was run with an inter-framing time between images of $\Delta t=480 \mathrm{~ns}$. The calculated velocity fields from the two cameras were stitched together using a convolution with an adaptive Gaussian window [42] that overlapped by $7.5 \%$, which results in an effective field of view of $10 D \times 2.2 D$.

A multigrid cross-correlation digital particle image velocimetry (MCCDPIV) algorithm [41] with an adaptive interrogation window technique was used. The adaptive interrogation window iteratively reduces an initial window size of $\mathrm{IW}_{0}$ to a final size of $I_{1}$, which were 64 pixels and 16 pixels, respectively, with a 50\% overlap. The maximum resolvable velocity was estimated based on a displacement of $25 \%$ of the initial interrogation window size. The minimum resolvable velocity was based on a conservative estimate of the minimum resolvable displacement as $0.1 \pm 0.06$ pixels ( $95 \%$ confidence level) [41]. Erroneous vectors were identified by a dynamic mean value operator and replaced by interpolation with immediate neighbours. Table 2 summarises the key PIV parameters.

\section{Flow Velocity Decomposition}

The fluctuating velocity variables are commonly calculated using the Reynolds decomposition. In screeching jets, a triple decomposition of flow variables [43] is more appropriate as it decouples the well organised large-scale coherent motion from the random smalland large-scale turbulence. The triple decomposition is defined as

$$
V_{i}(y, t)=\bar{V}_{i}(y)+v_{i}^{c}(y, t)+v_{i}^{\prime}(y, t)
$$

with the mean, coherent, and random components denoted by $\overline{\boldsymbol{V}}_{i}, \boldsymbol{v}_{i}^{\mathrm{c}}$, and $\boldsymbol{v}_{i}^{\prime}$, respectively. The flow variables are decomposed so that the sound, and its source, correspond to 
the fluctuating parts of the flow. The mean is steady, hence, radiates no sound, while the coherent and random components are considered to consist of the fluctuations that contribute only to the screech and broadband noise, respectively. The delineation between the contributions to screech and broadband noise neglects the large-scale mixing noise that is generated by the coherent fluctuations. The broadband noise mechanisms are studied in terms of the velocity fluctuations by filtering the coherent component associated with the screech tones. The coherent velocity fluctuations contain the high-energy large-scale periodic motion associated with the helical C instability screech mode, which is dominant for both jet conditions considered.

The coherent velocity fluctuations are determined using a snapshot POD method as outlined in Tan et al. [18]. The POD-based triple decomposition involves identifying the POD modes that sufficiently capture the periodic large-scale coherent motion present in the jet. Tan et al. identified through a spectral analysis that the periodic motion is well captured by the leading pair of POD modes for the LTRAC jet cases considered in this study. The velocity fluctuations contributing to the helical $\mathrm{C}$ screech instability mode are filtered by subtracting the coherent velocity fluctuations from Eq.(16), and thus their ability to generate noise. A shock capturing technique was not considered for the discontinuous behaviour across shocked regions in the potential core [44] as the fluctuations in the shear layer are more relevant in the consideration of BBSAN and mixing noise.

The generation of broadband noise includes contributions from all coherent structures, as well as all scales of turbulence, but these contributions are not necessarily periodic in nature. The POD analysis does not remove all structures at those scales, but only those that are periodic within the spatial domain. Thus, the reconstructed random velocity fluctuations consist of all structures and scales except the periodic large-scale coherent structures attributed to the screech tones, while the non-periodic structures at this wavelength remain. The fluctuations without the periodic self-forcing component can be used as input to an acoustic analogy. The random velocity fluctuations are expected to retain any tone-induced turbulence amplification effects [45] as they are expected to manifest in 
the smaller scales of turbulence [46].

Tan et al. [18] applied the method to demonstrate that the second-order integral length scales exhibited a similar scaling with the shear layer thickness to sub- and supersonic nonscreeching jet experiments $[30,47]$ when the coherent velocity fluctuations are filtered. It should be noted that the absolute values differed. The reliability of the POD-based method for studying the integral length scales of the random velocity fluctuations was assessed through a sensitivity analysis [18]. The analysis methodically reduced the resolution and SNR of the PIV velocity field before performing the POD analysis and integral length scale calculations. The error relative to calculations using the full resolution data is less than $1 \%$ when there are at least 3000 vector fields and a SNR greater than 500, while the error is approximately $1.5 \%$ when the PIV mesh is down-sampled to $146 \times 800$ vectors. The sensitivity of the POD analysis and length scale calculations to the sample size and rate was found to fall within the ranges predicted in similar studies [18,48-50]. The amplification of the random velocity fluctuations due to the presence of screech is expected to be on the order of the uncertainty in the POD analysis [18].

\section{Correlation Analysis}

The flow variables relevant for modelling the BBSAN and mixing noise sources are calculated directly from the spatial turbulence statistics of the PIV measurements. The measurements have been examined by Markesteijn et al. [28], where it was demonstrated that the velocity correlations is consistent with the quasi-normality hypothesis. The integral length scales are evaluated with an upper limit of integration that is defined by

$$
r^{v}(\boldsymbol{x}, \boldsymbol{\eta}=\Lambda, \tau=0)=0.1
$$

A cut-off criterion of 0.1 is used to avoid the low-correlation noise and contributions from negative correlations introduced by the screech mechanism. Tan et al. [18] found that there are negligible differences in magnitude when the zero crossing is used. 
The functional scales that define the models depend on the mean turbulence kinetic energy $k$ and dissipation rate $\epsilon$, which are not directly available from the measurements. The mean turbulence kinetic energy and dissipation rate are respectively given by

$$
k=\frac{1}{2}\left\langle v_{i}^{\prime} v_{i}^{\prime}\right\rangle \text { and } \epsilon=v\left\langle\frac{\partial v_{i}^{\prime}}{\partial x_{k}} \frac{\partial v_{i}^{\prime}}{\partial x_{k}}\right\rangle
$$

where $v$ is taken to be the kinematic viscosity of air at normal conditions. The accuracy in the dissipation rate calculation is determined by the resolution of the PIV measurements. To estimate the mean turbulence kinetic energy and dissipation rate, axisymmetric mean flow assumptions are applied such that

$$
\begin{aligned}
\left\langle v_{2}^{\prime} v_{2}^{\prime}\right\rangle & \approx\left\langle v_{3}^{\prime} v_{3}^{\prime}\right\rangle \\
\left\langle\frac{\partial v_{2}^{\prime}}{\partial x_{j}} \frac{\partial v_{2}^{\prime}}{\partial x_{j}}\right\rangle & \approx\left\langle\frac{\partial v_{3}^{\prime}}{\partial x_{j}} \frac{\partial v_{3}^{\prime}}{\partial x_{j}}\right\rangle \\
\left\langle\frac{\partial v_{i}^{\prime}}{\partial x_{2}} \frac{\partial v_{i}^{\prime}}{\partial x_{2}}\right\rangle & \approx\left\langle\frac{\partial v_{i}^{\prime}}{\partial x_{3}} \frac{\partial v_{i}^{\prime}}{\partial x_{3}}\right\rangle
\end{aligned}
$$

The integral scales are related to the local turbulence scales via proportionality coefficients. The proportionality coefficients $c_{L^{(\phi)}}$ are expressed as

$$
c_{L^{(\phi)}}=\frac{L^{(\phi)}}{\hat{L}^{(\phi)}}
$$

Evaluating Eq. (20) from the 2D PIV measurements yields proportionality coefficients for both the axial and transverse velocities. Only the symmetric tensor components are considered as they have the largest statistical significance in axisymmetric round unheated jets $[13,28]$. The RMSE associated with the proportionality coefficients is expressed as a percentage of the local integral length scale value such that

$$
\text { RMSE }=\frac{\sqrt{\overline{\left(L_{i}-\hat{L}_{i}\right)^{2}}}}{L_{i}}
$$




\section{Results and Discussion}

\section{A. Flow-Fields}

\section{Mean Velocity Statistics}
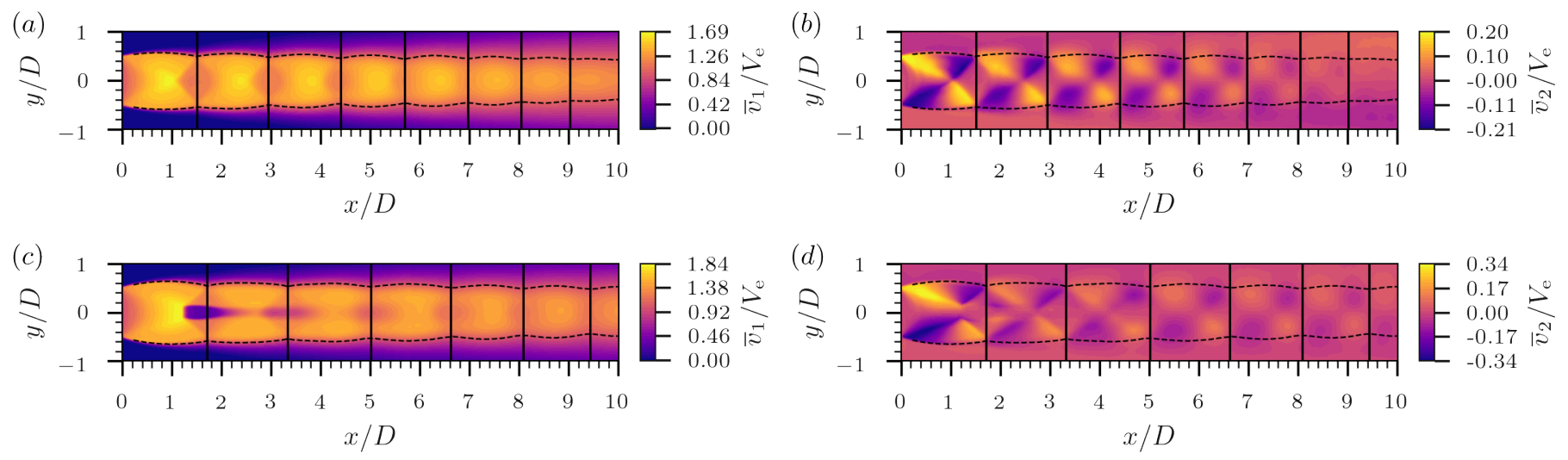

Figure 2. Contours of the mean velocity for $M_{\mathrm{j}}$ of $(\mathbf{a}, \mathbf{b}) 1.45$ and $(\mathbf{c}, \mathbf{d}) 1.59$

The mean axial and transverse velocity fields for $M_{\mathrm{j}}=1.45$ and 1.59 are shown in Fig.2. The values are normalised by the exit velocity with the sonic line and axial positions of the shock reflection points superimposed to illustrate the overall flow structure. The same shock reflection points are overlaid in subsequent plots when relevant. The sonic line, which is indicated by the overlaid dashed horizontal lines, is based on the local Mach number. The local Mach number is determined from the PIV measurements by assuming the total temperature is constant and equal to the temperature in the plenum chamber [30]. The jet cores have a supersonic region that is demarcated by the sonic line. A quasi-periodic shock cell structure is evident in the supersonic regions of the jets. The vertical lines correspond to the axial locations of the intersection of the shock tips with the high-speed side of the shear layer, which are determined by setting the mean axial velocity gradient $\partial \bar{V}_{1} / \partial x$ to zero. The shock spacing and strength are proportional to the Mach number. The subsonic region and internal shear layer that is evident in the core of the $M_{\mathrm{j}}=1.59$ jet is due to a Mach disk near $x / D=1$ [37]. 
2. Mean Turbulence Kinetic Energy and Dissipation Rate
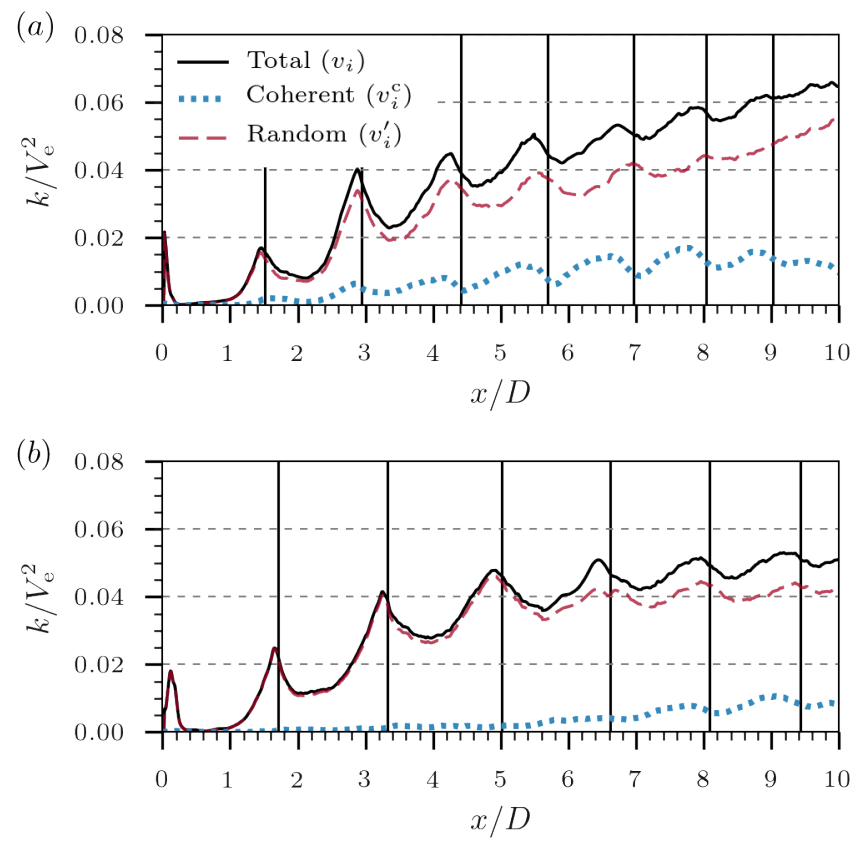

Figure 3. Turbulence kinetic energy along the nozzle lip line $(y / D=0.5)$ for $M_{j}$ of (a) 1.45 and (b) 1.59
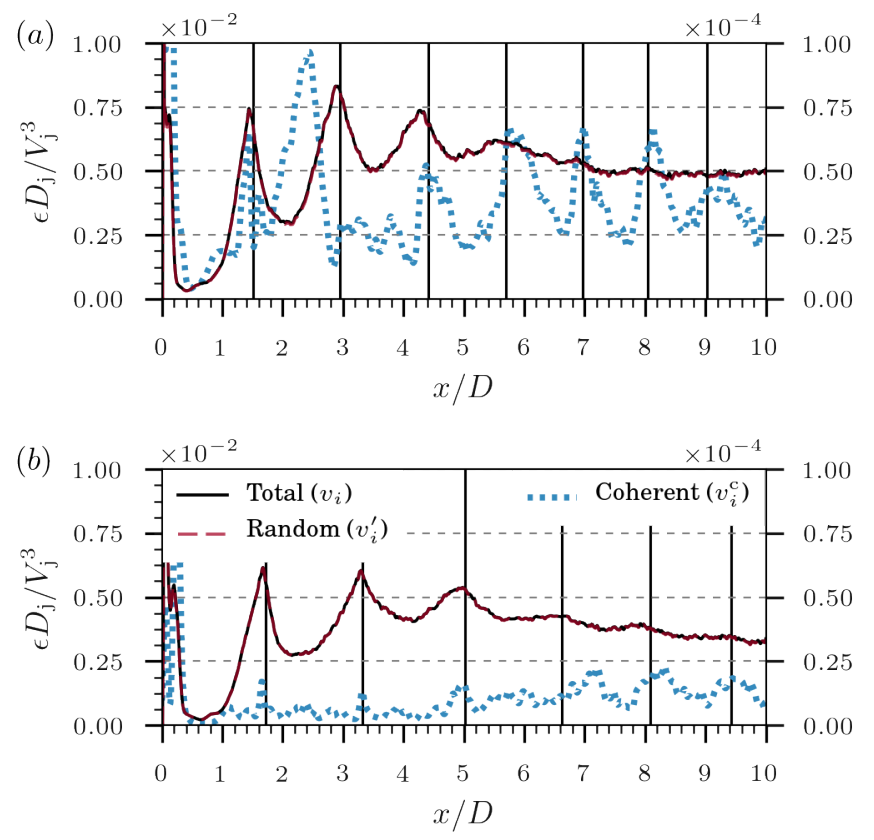

Figure 4. Turbulence dissipation rate organised in the same manner as Fig.3 with the coherent component corresponding to the axis on the right

The mean turbulence kinetic energy and dissipation rate that are used to calculate the 
local turbulence scales along the nozzle lip line are shown in Fig. 3 and 4. The mean turbulence statistics calculated from the total, coherent, and random velocity fluctuations are shown. The dissipation rate of the coherent velocity fluctuations is presented as the axis on the right of Fig. 4. The energy contributions from the random and coherent fluctuations are equivalent to the total turbulence kinetic energy. Thus, the amplitude of the turbulence kinetic energy is reduced when the high-energy large-scale periodic fluctuations that are associated with the screech tones are filtered. Consequently, some large-scale fluctuations contributing to the BBSAN are also removed.

The turbulence kinetic energy of both velocity components is small for $x / D<1$, which suggests the near nozzle region where the shear layer is thin does not contribute significantly to the radiated noise. Additionally, the uncertainty in the near nozzle region is expected to be larger due to limits in the resolution of the measurements. The kinetic energy of the random and coherent fluctuations are approximately in anti-phase, which is clearest at the shock reflection points for the $M_{j}=1.45$ case in Fig. 3(a). At the shock reflection points, the energy of the coherent velocity fluctuations decreases while the energy of the random velocity fluctuations increases. The relative changes in energy is reflected in the dissipation rate of the coherent velocity fluctuations shown in Fig. 4 as the energy is transferred to the random velocity fluctuations. The trends observed in the dissipation rate reflects the conservation of energy that is transferred from the large scales of motion to the small scales.

\section{B. Length Scales}

The second-order streamwise integral length scales that are important for modelling the BBSAN in its dominant propagation direction $\left(90^{\circ}\right.$ angle to the jet flow) depend on the transverse velocity fluctuations. The corresponding length scales along the nozzle lip line for $M_{j}=1.45$ and 1.59 are shown in Fig. 5. The integral scale components $L_{11}^{(2)}, L_{11}^{(2)}$, and $L_{22}^{(2)}$ are not shown as they have similar trends. The functional scales (symbols) are scaled so that they approximately overlap the integral length scales. The streamwise domain is 

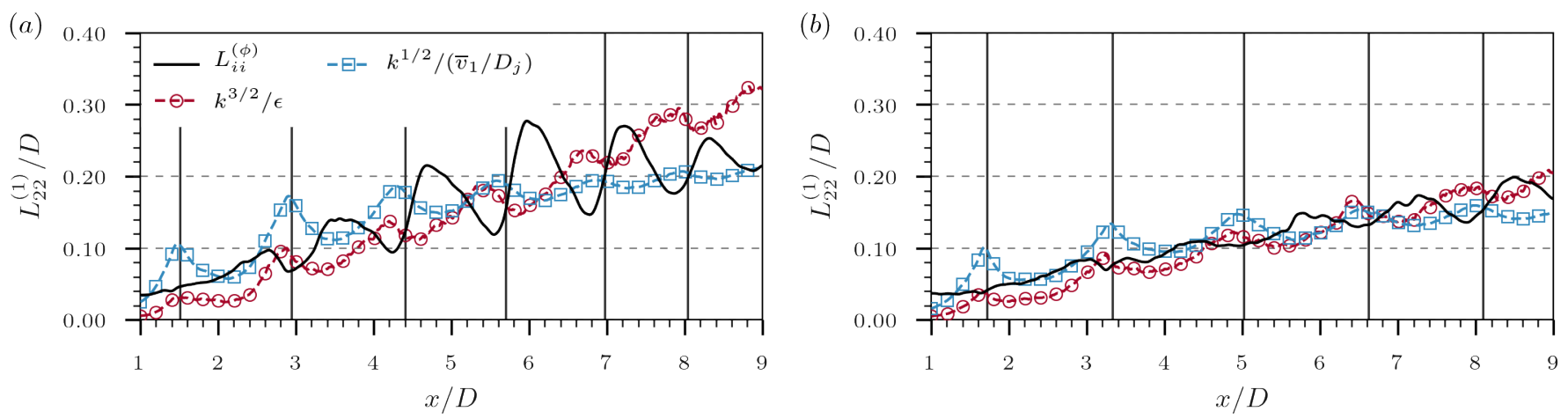

Figure 5. Streamwise second-order transverse length scales along the nozzle lip line $(y / D=0.5)$ for $M_{\mathrm{j}}$ of (a) 1.45 and (b) 1.59
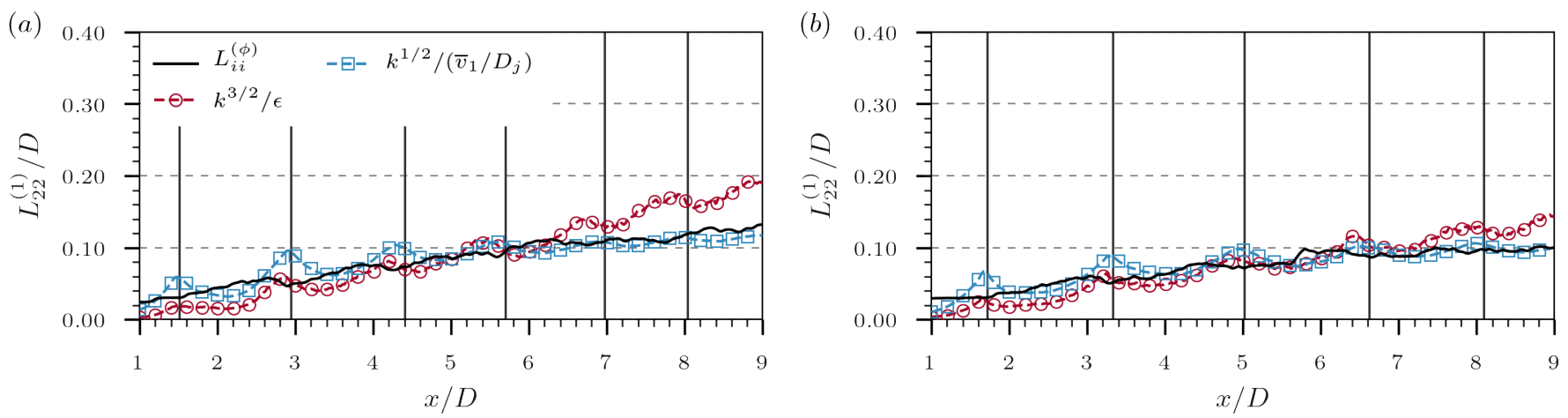

Figure 6. Streamwise second-order random axial length scales along the nozzle lip line $(y / D=0.5)$ for $M_{\mathrm{j}}$ of (a) 1.45 and (b) 1.59
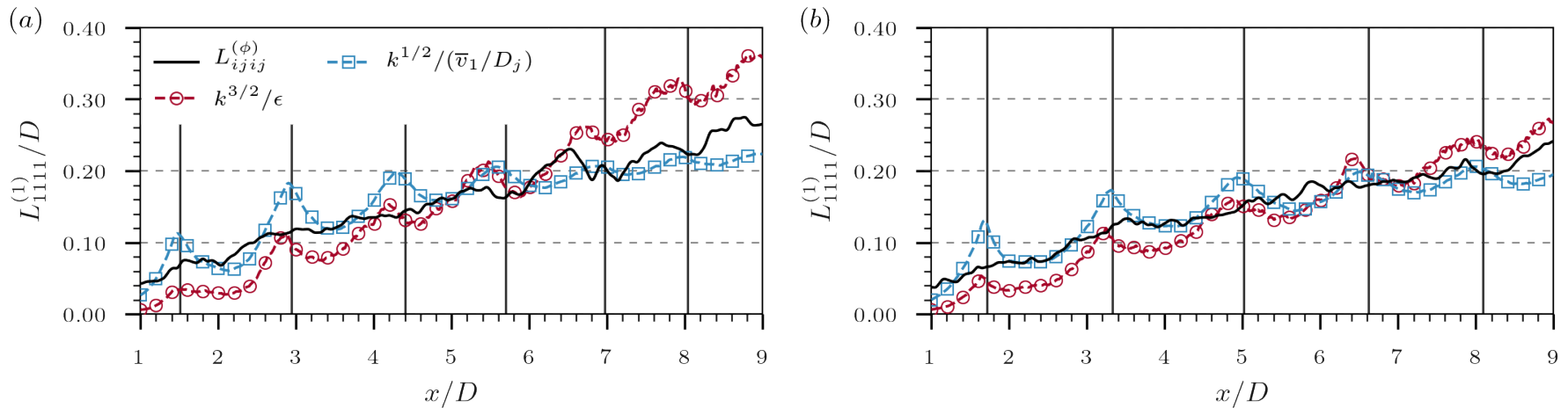

Figure 7. Streamwise fourth-order axial length scales along the nozzle lip line $(y / D=0.5)$ for $M_{\mathrm{j}}$ of (a) 1.45 and (b) 1.59 

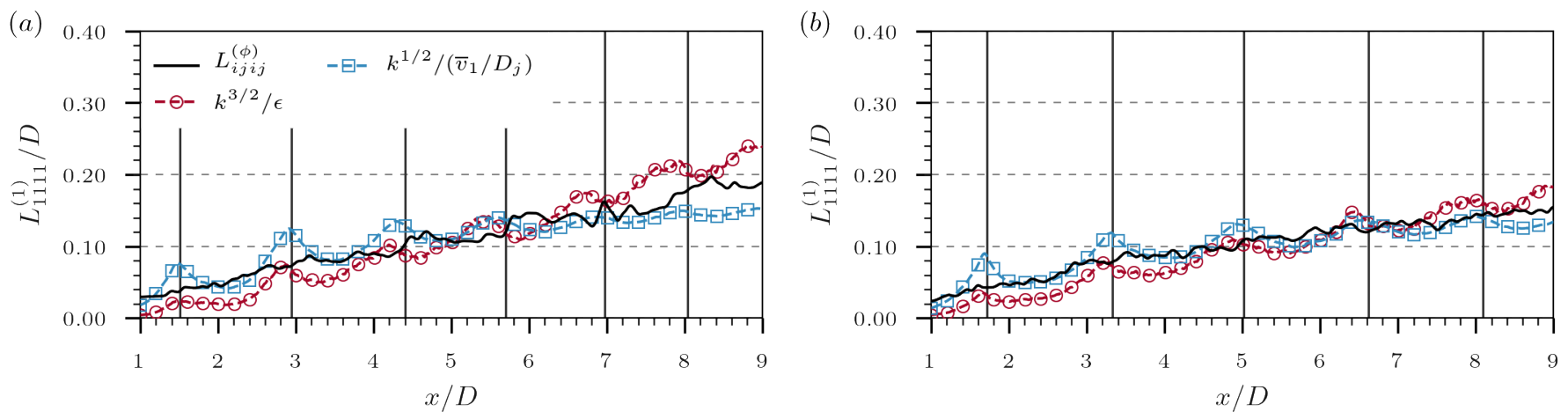

Figure 8. Streamwise fourth-order random axial length scales along the nozzle lip line $(y / D=0.5)$ for $M_{\mathrm{j}}$ of (a) 1.45 and (b) 1.59

restricted to $1<x / D<9$ as the near-nozzle region where the energy is small (Fig. 3) is not expected to contribute significantly to the radiated noise. The downstream values are truncated as the integral scales are underestimated at the boundaries of the measurement domain.

There is reasonable agreement between the gradients of the integral and functional scales. The integral scales have a linear trend that is modulated with a period corresponding to the shock cell spacing. The functional scales undergo a similar modulation that is out of phase with the integral scales such that they are locally small and large, respectively. The disagreement is indicative of the limits in the accuracy of linear models such as the ones considered here. The disagreement was found to be consistent with the integral scales $L_{11}^{(1)}, L_{11}^{(2)}$, and $L_{22}^{(2)}$.

The second-order streamwise integral and functional length scales calculated from the random transverse velocity fluctuations are shown in Fig.6. The value of the random integral length scales is reduced compared to the length scales of the total velocity fluctuations due to the filtering of the high-energy large-scale periodic turbulence. The length scales of the random velocity fluctuations are still modulated by the shock cells, though much less so than when the large-scale coherent periodic motion is included. The functional scales are comparable to Fig. 5 due to only a slight reduction in the mean turbulence kinetic energy for $x / D>4$, while the turbulence dissipation rate is relatively unchanged. 
The fourth-order length scales that characterise the mixing noise sources are shown in Fig.7 and 8, which correspond to the total and random velocity fluctuations, respectively. Thus, compared to the transverse velocity auto-correlation scales shown in Fig. 6, the fourth-order correlation scales correspond to the streamwise velocity component. The fourth-order integral scales have a linear trend without any significant modulation. The amplitude of the fourth-order components is reduced relative to the second-order scales, which is indicative of the range of energy containing turbulence scales that contribute to the radiated mixing noise. The reduction in amplitude when the large-scale periodic coherent fluctuations are filtered is also observed in the fourth-order length scales.

\section{Proportionality Coefficients}

Table 3. Summary of the proportionality coefficients for the second-order length scales

\begin{tabular}{|c|c|c|c|c|c|c|c|c|}
\hline & \multicolumn{4}{|c|}{ Total fluctuations } & \multicolumn{4}{|c|}{ Random fluctuations } \\
\hline & $M_{j}=1.45$ & $M_{\mathrm{j}}=1.59$ & $\mu\left(c_{L}\right)$ & $\Delta\left(c_{L}\right)$ & $M_{\mathrm{j}}=1.45$ & $M_{\mathrm{j}}=1.59$ & $\mu\left(c_{L}\right)$ & $\Delta\left(c_{L}\right)$ \\
\hline \multicolumn{9}{|c|}{$k^{3 / 2} / \epsilon:$} \\
\hline $\mathcal{C}_{L_{11}^{(1)}}$ & $0.67 \pm 0.19$ & $0.65 \pm 0.11$ & 0.66 & $3.03 \%$ & $0.62 \pm 0.14$ & $0.61 \pm 0.08$ & 0.62 & $1.63 \%$ \\
\hline$c_{L_{11}^{(2)}}$ & $0.40 \pm 0.08$ & $0.33 \pm 0.03$ & 0.37 & $19.2 \%$ & $0.29 \pm 0.03$ & $0.28 \pm 0.01$ & 0.29 & $3.51 \%$ \\
\hline$C_{L_{22}^{(1)}}$ & $0.42 \pm 0.15$ & $0.31 \pm 0.05$ & 0.37 & $30.1 \%$ & $0.29 \pm 0.06$ & $0.27 \pm 0.02$ & 0.28 & $7.14 \%$ \\
\hline$C_{L_{22}^{(2)}}$ & $0.53 \pm 0.20$ & $0.40 \pm 0.06$ & 0.47 & $28.0 \%$ & $0.28 \pm 0.05$ & $0.26 \pm 0.05$ & 0.27 & $7.41 \%$ \\
\hline \multicolumn{9}{|c|}{$k^{1 / 2} /\left(\bar{V}_{1} / D_{\mathrm{j}}\right)$} \\
\hline$c_{L_{11}^{(1)}}$ & $1.18 \pm 0.21$ & $1.32 \pm 0.17$ & 1.25 & $11.2 \%$ & $0.92 \pm 0.13$ & $0.94 \pm 0.11$ & 0.93 & $2.15 \%$ \\
\hline$c_{L_{11}^{(2)}}$ & $0.72 \pm 0.14$ & $0.67 \pm 0.09$ & 0.70 & $7.19 \%$ & $0.43 \pm 0.08$ & $0.45 \pm 0.06$ & 0.44 & $4.55 \%$ \\
\hline $\mathcal{C}_{L_{22}^{(1)}}$ & $0.74 \pm 0.21$ & $0.64 \pm 0.12$ & 0.69 & $14.5 \%$ & $0.44 \pm 0.07$ & $0.42 \pm 0.06$ & 0.43 & $4.65 \%$ \\
\hline$c_{L_{22}^{(2)}}$ & $0.92 \pm 0.19$ & $0.81 \pm 0.12$ & 0.87 & $12.7 \%$ & $0.42 \pm 0.08$ & $0.41 \pm 0.06$ & 0.42 & $2.41 \%$ \\
\hline
\end{tabular}

The integral and functional length scales are compared to evaluate the proportionality coefficients given by Eq. (20). The mean coefficient values of the dominant second- and fourth-order length scale components along the nozzle lip line $(y / D=0.5)$ for $1<x / D<9$ are summarised with the RMS dispersion error in Table 3 and 4, respectively. The mean value and relative discrepancy across both jet conditions are denoted by $\mu\left(c_{L}\right)$ and $\Delta\left(c_{L}\right)$, 
Table 4. Summary of the proportionality coefficients for the fourth-order length scales

\begin{tabular}{|c|c|c|c|c|c|c|c|c|}
\hline & \multicolumn{4}{|c|}{ Total fluctuations } & \multicolumn{4}{|c|}{ Random fluctuations } \\
\hline & $M_{j}=1.45$ & $M_{j}=1.59$ & $\mu\left(c_{L}\right)$ & $\Delta\left(c_{L}\right)$ & $M_{j}=1.45$ & $M_{j}=1.59$ & $\mu\left(c_{L}\right)$ & $\Delta\left(c_{L}\right)$ \\
\hline \multicolumn{9}{|c|}{$k^{3 / 2} / \epsilon:$} \\
\hline $\mathcal{C}_{L_{1111}^{(1)}}$ & $0.42 \pm 0.10$ & $0.41 \pm 0.07$ & 0.42 & $2.41 \%$ & $0.39 \pm 0.08$ & $0.37 \pm 0.04$ & 0.38 & $5.26 \%$ \\
\hline$C_{L_{1111}^{(2)}}$ & $0.25 \pm 0.04$ & $0.23 \pm 0.03$ & 0.24 & $8.33 \%$ & $0.25 \pm 0.04$ & $0.23 \pm 0.02$ & 0.24 & $8.33 \%$ \\
\hline$c_{L_{2222}^{(1)}}$ & $0.21 \pm 0.07$ & $0.19 \pm 0.03$ & 0.20 & $10.0 \%$ & $0.21 \pm 0.05$ & $0.20 \pm 0.03$ & 0.21 & $4.88 \%$ \\
\hline$c_{L_{2222}^{(2)}}$ & $0.24 \pm 0.05$ & $0.22 \pm 0.04$ & 0.23 & $8.70 \%$ & $0.22 \pm 0.04$ & $0.21 \pm 0.02$ & 0.22 & $4.65 \%$ \\
\hline$C_{L_{1212}^{(1)}}$ & $0.29 \pm 0.08$ & $0.26 \pm 0.05$ & 0.28 & $10.9 \%$ & $0.25 \pm 0.06$ & $0.23 \pm 0.03$ & 0.24 & $8.33 \%$ \\
\hline$c_{L_{1212}^{(2)}}$ & $0.26 \pm 0.05$ & $0.24 \pm 0.03$ & 0.25 & $8.00 \%$ & $0.25 \pm 0.05$ & $0.23 \pm 0.03$ & 0.24 & $8.33 \%$ \\
\hline \multicolumn{9}{|c|}{$k^{1 / 2} /\left(\bar{V}_{1} / D_{\mathrm{j}}\right)$} \\
\hline $\mathcal{C}_{L_{1111}^{(1)}}$ & $0.74 \pm 0.11$ & $0.82 \pm 0.10$ & 0.78 & $10.3 \%$ & $0.59 \pm 0.11$ & $0.60 \pm 0.08$ & 0.60 & $1.68 \%$ \\
\hline $\mathcal{C}_{L_{1111}^{(2)}}$ & $0.45 \pm 0.08$ & $0.46 \pm 0.05$ & 0.46 & $2.20 \%$ & $0.37 \pm 0.06$ & $0.37 \pm 0.04$ & 0.37 & $0.00 \%$ \\
\hline$c_{L_{2222}^{(1)}}$ & $0.39 \pm 0.07$ & $0.37 \pm 0.05$ & 0.38 & $5.26 \%$ & $0.34 \pm 0.05$ & $0.32 \pm 0.03$ & 0.33 & $6.06 \%$ \\
\hline$c_{L_{2222}^{(2)}}$ & $0.45 \pm 0.09$ & $0.43 \pm 0.06$ & 0.44 & $4.55 \%$ & $0.33 \pm 0.05$ & $0.30 \pm 0.03$ & 0.32 & $9.52 \%$ \\
\hline $\mathcal{C}_{L_{1212}^{(1)}}$ & $0.51 \pm 0.10$ & $0.52 \pm 0.08$ & 0.52 & $1.94 \%$ & $0.37 \pm 0.06$ & $0.37 \pm 0.05$ & 0.37 & $0.00 \%$ \\
\hline$c_{L_{1212}^{(2)}}$ & $0.48 \pm 0.09$ & $0.48 \pm 0.06$ & 0.48 & $0.00 \%$ & $0.37 \pm 0.06$ & $0.37 \pm 0.04$ & 0.37 & $0.00 \%$ \\
\hline
\end{tabular}

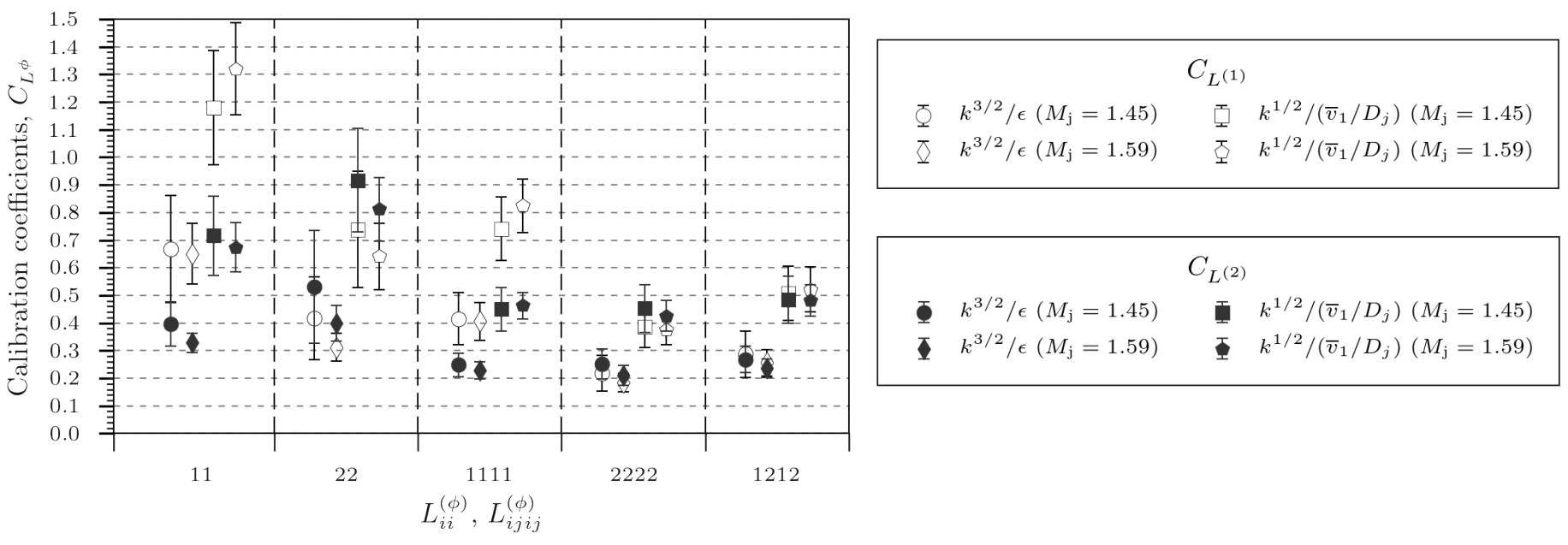

Figure 9. Proportionality coefficients for the total turbulence length scales

respectively. The mean coefficient values for the fourth-order scales are generally smaller compared to the second-order components, which is indicative of the turbulence scales that are considered to contribute to the mixing noise and BBSAN, respectively. The 


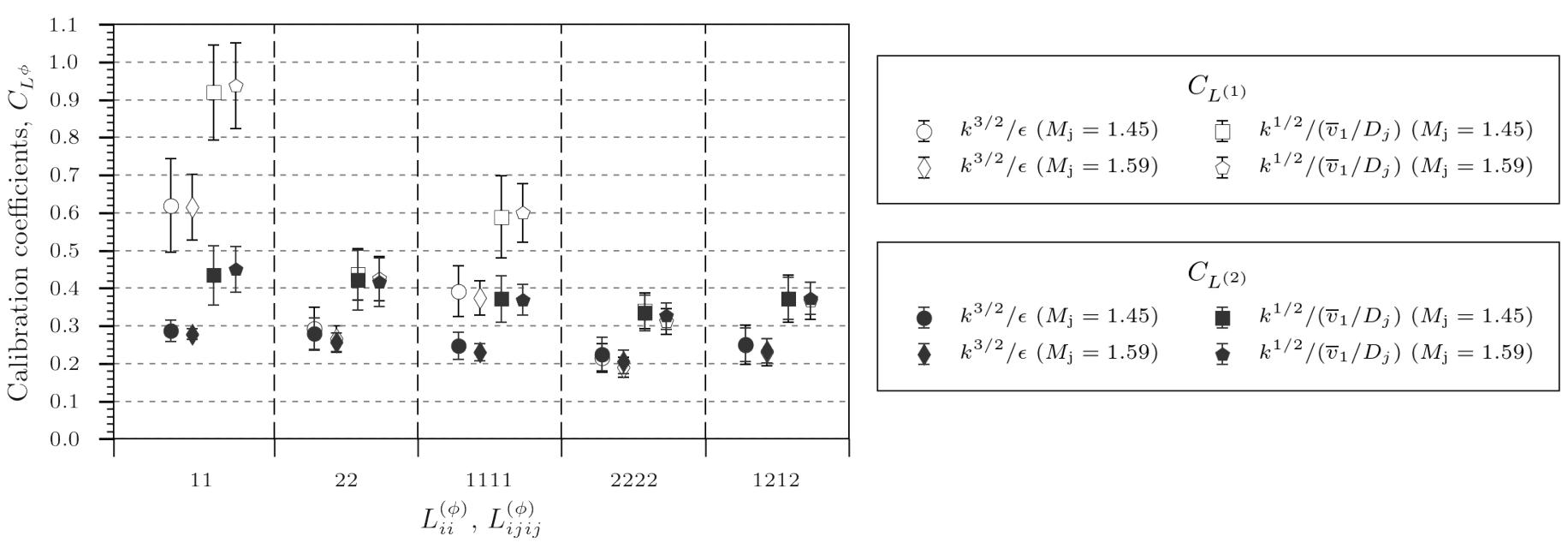

Figure 10. Proportionality coefficients for the random turbulence length scales

Table 5. Relative amplitudes of the fourth-order auto-correlation functions relative to $R_{1111}$ along $y / D_{\mathrm{j}}=$ 0.5

\begin{tabular}{lcccc}
\hline \hline & $M_{\mathrm{j}}$ & $x / D_{\mathrm{j}}$ & $R_{2222}$ & $R_{1212}$ \\
\hline LTRAC Jets: & & & & \\
Total fluctuations & 1.45 & 5.0 & 0.16 & 0.24 \\
& 1.59 & 5.0 & 0.17 & 0.24 \\
Random fluctuations & 1.45 & 5.0 & 0.15 & 0.22 \\
& 1.59 & 5.0 & 0.15 & 0.23
\end{tabular}

Literature:

$\begin{array}{lcccc}\text { Karabasov et al. [13] } & 0.75 & 4.0 & 0.34 & 0.37 \\ \text { Morris \& Zaman [51] } & & 6.0 & 0.24 & 0.31 \\ \text { Morris \& Zaman [19] } & 0.25 & 5.0 & 0.31 & 0.29 \\ \text { Leib \& Goldstein [52] } & 0.25 & 5.0 & 0.16 & 0.23\end{array}$

RMS dispersion error of the linear fit using the proportionality coefficients for each Mach number is included with the mean value for each jet condition. The mean coefficient value and percentage difference for both the Mach numbers considered are denoted by $\mu\left(C_{L}\right)$ and $\Delta\left(C_{L}\right)$, respectively. The percentage difference is calculated by

$$
\Delta\left(c_{L}\right)=\frac{\left|c_{L}\left(M_{\mathrm{j}}=1.45\right)-c_{L}\left(M_{\mathrm{j}}=1.59\right)\right|}{\mu\left(c_{L}\right)}
$$


such that larger relative discrepancies highlight the dependence of the low-order models on the operating condition of the jet.

The relative discrepancies between the coefficients at each Mach number are largest for the second-order length scales associated with the total velocity fluctuations, as can be seen from Table 3. The dependence on the Mach number is expected to be linked to the large modulation of the total length scales that is evident in Fig. 5. The fourth-order coefficients presented in Table 4 are not as sensitive to the mean jet conditions. The trends in the second- and fourth-order coefficients of the total integral length scales are evident in the scatter plot shown in Fig. 9, where the error bars are used to denote the RMS dispersion error for each jet condition. The dispersion error is significantly larger for the second-order coefficients, which is a result of the modulation of the integral length scales of the total velocity fluctuations observed in Fig.5. The dispersion error is smaller for the fourth-order models as the integral scales are not as strongly modulated, as can be seen in Fig.7.

The proportionality coefficients of the second- and fourth-order length scales of the random velocity fluctuations are shown in Fig. 10. Filtering the large-scale coherent periodic fluctuations reduces the relative Mach number discrepancies for the secondorder models. The effects of the filtering on the fourth-order models are not as large, with the relative Mach number discrepancies being comparable to those associated with the total velocity fluctuations. However, both the second- and fourth-order models have nearly constant coefficients for approximating the random integral length scales at both Mach numbers such that the markers for the streamwise and transverse components overlap.

The constant value of the proportionality coefficients for both jet conditions is promising for modelling the scales important for noise predictions. The filtered velocity fluctuations also lead to improved agreement between the integral scales and the low-order models as there is a smaller RMS dispersion error for all components and both jet conditions. Significant turbulence amplification effects due to the higher intensity screech tone 
at $M_{\mathrm{j}}=1.45$ are not evident in the proportionality coefficients when compared to the values for the other Mach number condition. Variations in the coefficient values are on the order of the RMS dispersion error. Given the weak dependence on Mach number and that the coefficients are typically fixed for predictions within a given operating regime, the values presented in Fig.10 are expected to be applicable to underexpanded non-screeching jets in a similar operating range.

An estimate for the anisotropy is obtained by comparing the streamwise axial coefficients $c_{L_{i j}^{(1)}}$ to the transverse coefficients $c_{L_{i j}^{(2)}}$. Anisotropy effects are clearly identified in Fig. 9 and 10 where the scales of the axial velocity fluctuations have the largest amplitudes. The relative magnitudes of the coefficients suggests that a suitable value to account for anisotropy in the second- and fourth-order length scales for $M_{\mathrm{j}}=1.45$ and 1.59 is $c_{L^{(2)}} \approx 0.5$ for both the turbulence dissipation and large eddy convection based models. The coefficient is slightly larger than previous implementations of the BBSAN model where a value of 0.3 was used for predicting the noise for jet conditions with and without screech $[3,4]$. The coefficient values were determined by matching the predicted noise to acoustic measurements for a single observer position and jet condition. The anisotropy is insignificant for the 22, 2222, and 1212 components as the streamwise and transverse length scale coefficients are approximately equal. These components have coefficients values that are approximately equal to those of $c_{L_{11}^{(2)}}$.

The amplitudes of the fourth-order length scale coefficients is consistent with the experimental and numerical results of Karabasov et al. [13] where a Gaussian mixing noise source model was fit to LES and RANS data. The fourth-order length scale coefficients were determined to be $c_{L_{1111}}=0.37$ for homogeneous and isotropic noise sources in an isothermal $M_{\mathrm{j}}=0.75$ axisymmetric jet. These coefficient values compare well with the current values determined directly from the PIV measurements, which have mean values of $c_{L_{1111}}=0.39$ and 0.37 for $M_{\mathrm{j}}=1.45$ and 1.59, respectively. Studies that determined the coefficients for $c_{L_{1111}}$ by matching noise predictions to far-field measurements yielded values of 0.13 and 0.78 , which were reported by Tam and Auriault [17], and Morris and 
Farassat [15], respectively. The fourth-order coefficients contrast the values for the secondorder coefficients used to scale the dissipation based turbulence scale used by Morris and Miller [3] for BBSAN were larger than the value Tam and Auriault [17] used in their prediction of fine-scale turbulence mixing noise.

In terms of the fourth-order Reynolds stress auto-correlation amplitudes $R_{i j k l}(y, 0,0)$, there are some discrepancies between the measurements of the LTRAC jets and the values determined by Karabasov et al. [13] from the LES data. The values of the amplitudes relative to the axial component $R_{1111}$ extracted from the LES data are larger than the current results. These results are summarised in Table 5 with values from similar studies. The fourth-order correlation amplitudes of the LTRAC jets agrees reasonably well with the single wire measurements of Morris and Zaman [19], and the analysis of experimental and LES data by Leib and Goldstein [52] (Table 5). The agreement is consistent for the total and random fluctuations at both Mach numbers considered. Inconsistencies are evident in the experiments conducted by Morris and Zaman $[19,51]$ where Mach 0.25 jets with low Reynolds numbers were analysed. These jets were made fully turbulent at the exit by placing a trip ring inside the nozzle. The use of a trip ring and low Reynolds number jets may have contributed to considerable variations in the relative amplitudes with position in the jet that were observed [19].

\section{Mean Square Error Analysis}
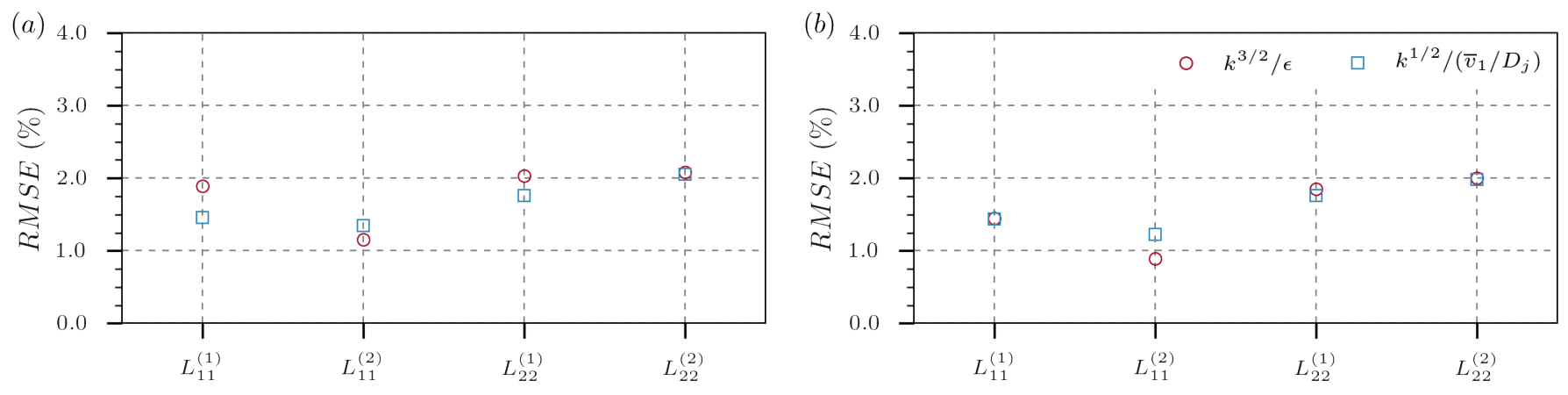

Figure 11. The RMSE of the second-order length scales for $M_{j}$ of (a) 1.45 and (b) 1.59 

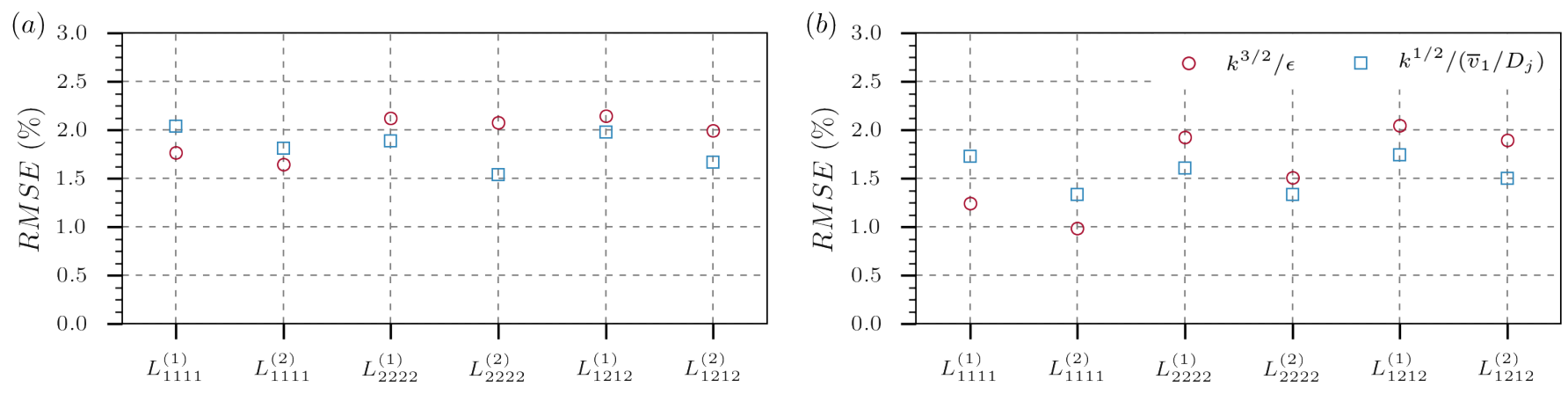

Figure 12. The RMSE of the fourth-order length scales for $M_{\mathrm{j}}$ of (a) 1.45 and (b) 1.59

The functional scales that best represent the underlying physics associated with each component of the integral length scales of the random velocity fluctuations are determined based on the RMSE. The RMSE for each of the second- and fourth-order random length scales are shown in Fig.11 and Fig.12, respectively. The RMSE is presented as a percentage of the local random turbulence integral length scale. The trends in the RMSE of the modelled second- and fourth-order length scales is consistent at both jet conditions. The RMSE of the length scales $L_{11}^{(2)}, L_{1111}^{(1)}$, and $L_{1111}^{(2)}$ tends to be smaller for the turbulence dissipation scale $k^{3 / 2} / \epsilon$, as can be seen in Fig. 11 and Fig. 12. The remaining length scales have errors that are minimised by using the large eddy convection scale $k^{1 / 2} /\left(\overline{V_{1}} / D_{\mathrm{j}}\right)$. This scale eliminates the turbulence dissipation term such that only the turbulence kinetic energy and mean axial velocity are required to consistently model the integral scales. The functional scales have errors less than 3\% for all components such that both loworder models provide an acceptable approximation of the integral length scales. This is promising since a $10 \%$ variation in the length scale coefficients is expected to result in a nominal error of less than 1\% in the predicted BBSAN [24] and mixing noise [13] spectra.

Distributions of the second- and fourth-order integral length scales along the nozzle lip line are shown in Fig. 13 and Fig. 14. The functional scale with the smallest RMSE for each integral scale component is shown, where the scales are evaluated with the proportionality coefficients in Fig.10. Overall, both local turbulence scale models provide a good approximation for the trend of the integral length scale distributions. The length 

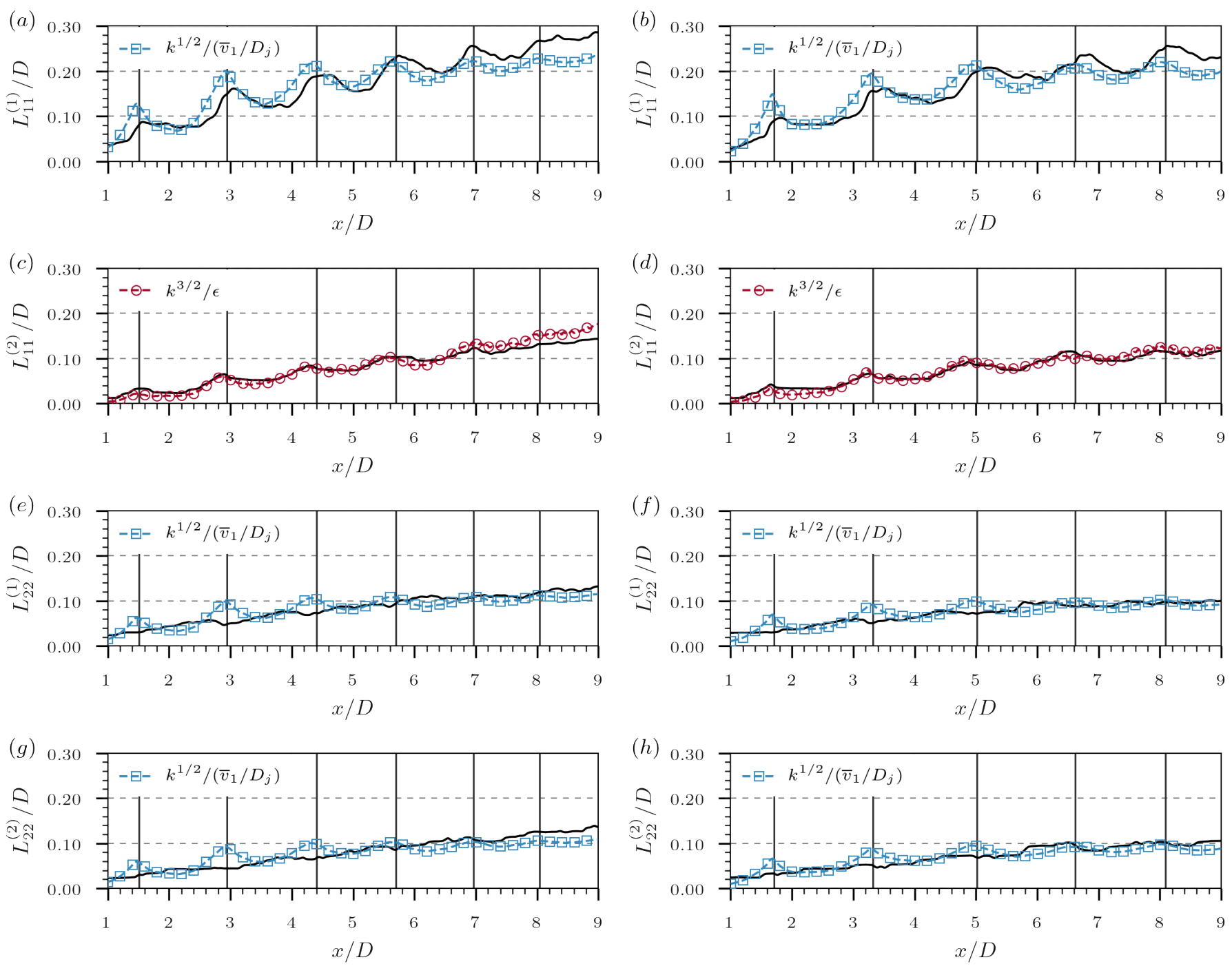

Figure 13. Distributions of the second-order random turbulence length scales for $M_{\mathrm{j}}$ of $(\mathbf{a}, \mathbf{c}, \mathbf{e}, \mathbf{g}) 1.45$ and $(\mathbf{b}, \mathbf{d}, \mathbf{f}, \mathbf{h}) 1.59$

scales $L_{11}^{(1)}$ and $L_{11}^{(2)}$ have excellent agreement with the functional scales with local maxima that are coincident with the shock reflection points. The modulation is not observed in the results of Tan et al. [18] as the distributions are along the centre of the shear layer, which is influenced by the oscillatory radial motion of the shock structures. The modulation for the length scales $L_{22}^{(1)}$ and $L_{22}^{(2)}$ is much weaker, which results in discrepancies at the shock reflection points. Discrepancies at the shock reflection points are more prevalent for the fourth-order components as the modulation of the integral length scales is much weaker, as can be seen in Fig. 14 . 


\section{Contribution of the Random Turbulence Scales}

Distributions along the nozzle lip line of the non-dimensional function calculated using the random velocity fluctuations are shown in Fig.15. The amplitude indicates the relative contribution of the time processes associated with the random turbulence and its scattering by the shock cells (Eq. (11) for $\alpha=2$ ). The distributions have values less than1 over the streamwise domain for both turbulence scales considered. In the framework of the mixed-scale model [4], this suggests that the noise associated with the dissipation of the turbulence dominates for the LTRAC jets. Consequently, the jet lip line area that is important for noise generation is dominated by the effects associated with the random velocity fluctuations. Importantly, the same conclusion follows from either using the standard turbulence dissipation scale or the large eddy convection scale model given by Eq. (14) and (15), respectively.

\section{Conclusions}

The second- and fourth-order turbulence length scales in screeching underexpanded unheated round jets with ideally expanded Mach numbers of 1.45 and 1.59 were studied. The integral length scales of the total velocity fluctuations were shown to have a linear trend that is modulated with a period corresponding to the shock cell spacing, but out of phase. A similar modulation was observed for functional scales that represent elements of various attempts to model BBSAN. The scales associated with the random velocity fluctuations were shown to have smaller amplitudes due to the filtering of the highenergy large-scale periodic coherent fluctuations. These trends were consistent for both the second- and fourth-order length scales. The fourth-order length scale components have smaller amplitudes relative to the second-order scales, which is indicative of the range of energy containing turbulence scales that contribute to the radiated mixing noise.

The mean value of the proportionality coefficients along the nozzle lip line that are important for modelling the broadband noise sources were presented. The relative discrepancies between the coefficients at each Mach number considered were largest for the 
second-order length scales of the total velocity fluctuations due to the strong modulation of the integral scales. The modulation of the integral scales also resulted in relatively large RMS dispersion errors for the linear relationship. The proportionality coefficients of the fourth-order length scales were less sensitive to the mean jet flow. This was also observed when considering the scales associated with only the random velocity fluctuations. The proportionality coefficients that directly relate the local turbulence scales to the integral scales of the random velocity fluctuations were shown to be independent of Mach number. Furthermore, anisotropy effects were found to be significant only in the axial 11 and 1111 components, which is consistent with results reported in the literature; however, there were discrepancies in the relative fourth-order auto-correlation amplitudes.

The turbulence dissipation and large eddy convection time based models were shown to approximate the integral length scales within $3 \%$ for $1<x / D<9$ at both jet conditions. The turbulence dissipation scale $k^{3 / 2} / \epsilon$ results in marginally smaller errors for $L_{11}^{(2)}, L_{1111}^{(1)}$, and $L_{1111}^{(2)}$. The remaining length scales are best represented by the large eddy convection scale $k^{1 / 2} /\left(\overline{V_{1}} / D_{\mathrm{j}}\right)$, which depends on the mean turbulence kinetic energy, mean axial velocity, ideally expanded exit diameter and Mach number, and ratio of specific heats. The ideally expanded jet diameter is a well-defined function of the jet flow so does not depend on the far-field acoustic variable. Therefore, an acoustic model that uses the suggested convection velocity scale for far-field noise predictions would be closed. The relatively small differences in the integral length scales and the functional scales are due to the effects associated with the random velocity fluctuations dominating along the nozzle lip line of the LTRAC jets. Despite minor differences between the turbulence dissipation and large eddy convection based scales, both provide an acceptable approximation of the integral length scales that are required for modelling the equivalent jet noise sources.

\section{Acknowledgments}

The authors would like to acknowledge the support of the Australian Research Council, the Research at Cloud Monash (R@CMon) National eResearch Collaboration Tools and 
Resources (NeCTAR) project funded by the Australia Commonwealth Government, and

The School of Engineering and Materials Sciences, Queen Mary University of London.

\section{References}

[1] Powell, A., "On the mechanism of choked jet noise," Proceedings of the Physical Society. Section B, Vol. 66, No. 12, 1953, pp. 1039. doi:10.1088/0370-1301/66/12/306.

[2] Tam, C. K. W. and Tanna, H. K., "Shock associated noise of supersonic jets from convergentdivergent nozzles," Journal of Sound and Vibration, Vol. 81, No. 3, 1982, pp. 337-358. doi:10.1016/0022-460X(82)90244-9.

[3] Morris, P. J. and Miller, S. A. E., "Prediction of broadband shock-associated noise using Reynolds-averaged Navier-Stokes computational fluid dynamics," AIAA journal, Vol. 48, No. 12, 2010, pp. 2931-2944. doi:10.2514/1.J050560.

[4] Kalyan, A. and Karabasov, S. A., "Broadband shock associated noise predictions in axisymmetric and asymmetric jets using an improved turbulence scale model," Journal of Sound and Vibration, Vol. 394, 2017, pp. 392-417. doi:10.1016/j.jsv.2017.01.027.

[5] Goldstein, M. E., “A generalized acoustic analogy,” Journal of Fluid Mechanics, Vol. 488, 2003, pp. 315-333. doi:10.1017/S0022112003004890.

[6] Lighthill, M. J., "On sound generated aerodynamically. I. General theory," Proceedings of the Royal Society of London A: Mathematical, Physical and Engineering Sciences, Vol. 211, The Royal Society, 1952, pp. 564-587. doi:10.1098/rspa.1952.0060.

[7] Freund, J. B., Lele, S. K., and Moin, P., "Calculation of the radiated sound field using an open Kirchhoff surface," AIAA journal, Vol. 34, No. 5, 1996. doi:10.2514/3.13167.

[8] Pilon, A. R. and Lyrintzis, A. S., "Development of an improved Kirchhoff method for jet aeroacoustics," AIAA journal, Vol. 36, No. 5, 1998, pp. 783-790. doi:10.2514/2.437.

[9] Williams, J. E. F. and Hawkings, D. L., "Sound generation by turbulence and surfaces in arbitrary motion," Philosophical Transactions of the Royal Society of London A: Mathematical, Physical and Engineering Sciences, Vol. 264, No. 1151, 1969, pp. 321-342. doi:10.1098/rsta.1969.0031.

[10] Freund, J., Lele, S., and Moin, P., "Numerical simulation of a Mach 1.92 turbulent jet and its sound field," AIAA journal, Vol. 38, No. 11, 2000, pp. 2023-2031. doi:10.2514/2.889.

[11] Miller, S. A. E., “Toward a Nonlinear Acoustic Analogy: Turbulence as a Source of Sound and Nonlinear Propagation," NASA TR-218706, 2015.

[12] Khavaran, A., Krejsa, E. A., and Kim, C. M., "Computation of supersonic jet mixing noise for an axisymmetric convergent-divergent nozzle," Journal of Aircraft, Vol. 31, No. 3, 1994, pp. 603-609. doi:10.2514/3.46537.

[13] Karabasov, S. A., Afsar, M. Z., Hynes, T. P., Dowling, A. P., McMullan, W. A., Pokora, C. D., Page, G. J., and McGuirk, J. J., "Jet noise: acoustic analogy informed by large eddy simulation," AIAA journal, Vol. 48, No. 7, 2010, pp. 1312-1325. doi:10.2514/1.44689.

[14] Miller, S. A. E., "Toward a comprehensive model of jet noise using an acoustic analogy," AIAA Journal, Vol. 52, No. 10, 2014, pp. 2143-2164. doi:10.2514/1.J052809. 
[15] Morris, P. J. and Farassat, F., "Acoustic analogy and alternative theories for jet noise prediction," AIAA journal, Vol. 40, No. 4, 2002, pp. 671-680. doi:10.2514/2.1699.

[16] Hunt, J., Moin, P., Lee, M., Moser, R., Spalart, P., Mansour, N., Kaimal, J., and Gaynor, E., "Cross correlation and length scales in turbulent flows near surfaces," Advances in Turbulence 2, Springer, 1989, pp. 128-134. doi:10.1007/978-3-642-83822-4.

[17] Tam, C. K. W. and Auriault, L., "Jet mixing noise from fine-scale turbulence," AIAA journal, Vol. 37, No. 2, 1999, pp. 145-153. doi:10.2514/2.691.

[18] Tan, D., Soria, J., Honnery, D., and Edgington-Mitchell, D., “Novel Method for Investigating Broadband Velocity Fluctuations in Axisymmetric Screeching Jets," AIAA Journal, Vol. 55, No. 7, 2017, pp. 2321-2334. doi:10.2514/1.J055606.

[19] Morris, P. J. and Zaman, K. B. M. Q., "Velocity measurements in jets with application to noise source modeling," Journal of sound and vibration, Vol. 329, No. 4, 2010, pp. 394-414. doi:10.1016/j.jsv.2009.09.024.

[20] Ribner, H.S., "The generation of sound by turbulent jets," Advances in applied mechanics, Vol. 8, No. 103-182, 1964, pp. 10. doi:10.1016/S0065-2156(08)70354-5.

[21] Azarpeyvand, M. and Self, R., "Improved jet noise modeling using a new time-scale," The Journal of the Acoustical Society of America, Vol. 126, No. 3, 2009, pp. 1015-1025. doi:10.1121/1.3192221.

[22] Kerhervé, F. and Fitzpatrick, J., "Measurement and analysis of the turbulent length scales in jet flows," Experiments in fluids, Vol. 50, No. 3, 2011, pp. 637-651. doi:10.1007/s00348-010-0957-2.

[23] Kamruzzaman, M., Lutz, T., Kraemer, E., and Wuerz, W., "On the length scales of turbulence for aeroacoustic applications," 17th AIAA/CEAS Aeroacoustics Conference (32nd AIAA Aeroacoustics Conference), 2011, p. 2734. doi:10.2514/6.2011-2734.

[24] Kalyan, A. and Karabasov, S. A., "Towards a Suitable Turbulence Length and Temporal Scale Model for Broadband Shock Associated Noise," 22nd AIAA/CEAS Aeroacoustics Conference, 2016, p. 2802. doi:10.2514/6.2016-2802.

[25] Khavaran, A. and Bridges, J., "Modelling of fine-scale turbulence mixing noise," Journal of Sound and Vibration, Vol. 279, No. 3, 2002, pp. 1131-1154. doi:10.1016/j.jsv.2003.11.054.

[26] Goldstein, M. and Leib, S., "The aeroacoustics of slowly diverging supersonic jets," Journal of Fluid Mechanics, Vol. 600, 2008, pp. 291-337. doi:10.1017/S0022112008000311.

[27] Morris, P., "Jet noise: a perspective on recent developments and future directions," Société Française d'Acoustique, Acoustics 2012, April 2012.

[28] Markesteijn, A. P., Semiletov, V., Karabasov, S. A., Tan, D. J., Wong, M., Honnery, D., and Edgington-Mitchell, D., "Supersonic Jet Noise: an Investigation into Noise Generation Mechanisms using Large Eddy Simulation and High-Resolution PIV Data," 23rd AIAA/CEAS Aeroacoustics Conference, 2017, p. 3029. doi:10.2514/6.2017-3029.

[29] O’Neill, P. L., Nicolaides, D., Honnery, D. R., and Soria, J., “Autocorrelation functions and the determination of integral length with reference to experimental and numerical data," 15th Australasian Fluid Mechanics Conference The University of Sydney, Sydney, Australia, 2004, pp. 13-17. 
[30] André, B., Castelain, T., and Bailly, C., "Investigation of the mixing layer of underexpanded supersonic jets by particle image velocimetry," International Journal of Heat and Fluid Flow, Vol. 50, 2014, pp. 188-200. doi:10.1016/j.ijheatfluidflow.2014.08.004.

[31] Tan, D. J., Soria, J., Honnery, D. R., and Edgington-Mitchell, D. M., "An Investigation of the Turbulent Integral Length Scales in an Underexpanded Axisymmetric Jet," Australian Conference on Laser Diagnostics in Fluid Mechanics and Combustion, Monash University Publishing, 2015, pp. 173-178.

[32] Tam, C. K. W., "Broadband shock-associated noise of moderately imperfectly expanded supersonic jets," Journal of Sound and Vibration, Vol. 140, No. 1, 1990, pp. 55-71. doi:10.1016/0022460X(90)90906-G.

[33] Miller, S. A. E., "The scaling of broadband shock-associated noise with increasing temperature," International Journal of Aeroacoustics, Vol. 14, No. 1-2, 2015, pp. 305-326. doi:10.1260/1475472X.14.1-2.305.

[34] Bridges, J. E. and Wernet, M. P., "Measurements of Turbulent Convection Speeds in Multistream Jets Using Time-Resolved PIV," 23rd AIAA/CEAS Aeroacoustics Conference, 2017, p. 4041.

[35] Frisch, U., Turbulence: the legacy of AN Kolmogorov, Cambridge university press, 1995. doi:10.1007/BF02508484.

[36] Edgington-Mitchell, D. M., Oberleithner, K., Honnery, D. R., and Soria, J., “Coherent structure and sound production in the helical mode of a screeching axisymmetric jet," Journal of Fluid Mechanics, Vol. 748, June 2014, pp. 822-847. doi:10.1017/jfm.2014.173.

[37] Edgington-Mitchell, D. M., Honnery, D. R., and Soria, J., "The underexpanded jet Mach disk and its associated shear layer," Physics of Fluids (1994-present), Vol. 26, No. 9, 2014, pp. 96-101. doi:10.1063/1.4894741.

[38] André, B., Castelain, T., and Bailly, C., "Effect of a tab on the aerodynamical development and noise of an underexpanded supersonic jet," Comptes Rendus Mécanique, Vol. 341, No. 9-10, 2013, pp. 659-666. doi:10.1016/j.crme.2013.08.001.

[39] Mitchell, D. M., Honnery, D. R., and Soria, J., "Near-field structure of underexpanded elliptic jets," Experiments in fluids, Vol. 54, No. 7, 2013, pp. 1-13. doi:10.1007/s00348-013-1578-3.

[40] Mitchell, D. M., Honnery, D. R., and Soria, J., "Particle relaxation and its influence on the particle image velocimetry cross-correlation function," Experiments in fluids, Vol. 51, No. 4, 2011, pp. 933-947. doi:10.1007/s00348-011-1116-0.

[41] Soria, J., "An investigation of the near wake of a circular cylinder using a video-based digital cross-correlation particle image velocimetry technique," Experimental Thermal and Fluid Science, Vol. 12, No. 2, 1996, pp. 221-233. doi:10.1016/0894-1777(95)00086-0.

[42] Agüí, J. C. and Jimenez, J., "On the performance of particle tracking," Journal of fluid mechanics, Vol. 185, December 1987, pp. 447-468. doi:10.1017/S0022112087003252.

[43] Hussain, A. K. M. F. and Reynolds, W. C., "The mechanics of an organized wave in turbulent shear flow," Journal of Fluid Mechanics, Vol. 41, No. 02, 1970, pp. 241-258. doi:10.1017/S0022112070000605. 
[44] Hall, K. C., Thomas, J. P., and Dowell, E. H., “Proper orthogonal decomposition technique for transonic unsteady aerodynamic flows," AIAA journal, Vol. 38, No. 10, 2000, pp. 1853-1862. doi:10.2514/2.867.

[45] Morris, P., "Broadband jet noise amplification by a pure tone excitation," NASA CR-163269, 1980.

[46] Ahuja, K. K., Lepicovsky, J., Tam, C. K. W., Morris, P. J., and Burrin, R. H., “Tone-excited jet: Theory and experiments," NASA CR-3538, 1982.

[47] Fleury, V., Bailly, C., Jondeau, E., Michard, M., and Juvé, D., “Space-time correlations in two subsonic jets using dual particle image velocimetry measurements," AIAA journal, Vol. 46, No. 10, 2008, pp. 2498-2509. doi:10.2514/1.35561.

[48] Glauser, M. N. and George, W. K., "Application of multipoint measurements for flow characterization," Experimental Thermal and Fluid Science, Vol. 5, No. 5, 1992, pp. 617-632. doi:10.1016/0894-1777(92)90018-Z.

[49] Tinney, C. E., Glauser, M. N., and Ukeiley, L. S., "Low-dimensional characteristics of a transonic jet. Part 1. Proper orthogonal decomposition," Journal of Fluid Mechanics, Vol. 612, October 2008, pp. 107-141. doi:10.1017/S0022112008002978.

[50] Tinney, C. E., "Proper grid resolutions for the proper basis." AIAA 47th Aerospace Sciences Meeting and Exhibit, Orlando, Florida, USA, AIAA paper, Vol. 68, 2009. doi:10.2514/6.2009-68.

[51] Morris, P. and Zaman, K., "Two component velocity correlations in jets and noise source modeling," 16th AIAA/CEAS Aeroacoustics Conference, 2010, p. 3781. doi:10.2514/6.2010-3781.

[52] Leib, S. J. and Goldstein, M. E., "Hybrid source model for predicting high-speed jet noise," AIAA journal, Vol. 49, No. 7, 2011, pp. 1324-1335. doi:10.2514/1.J050707. 

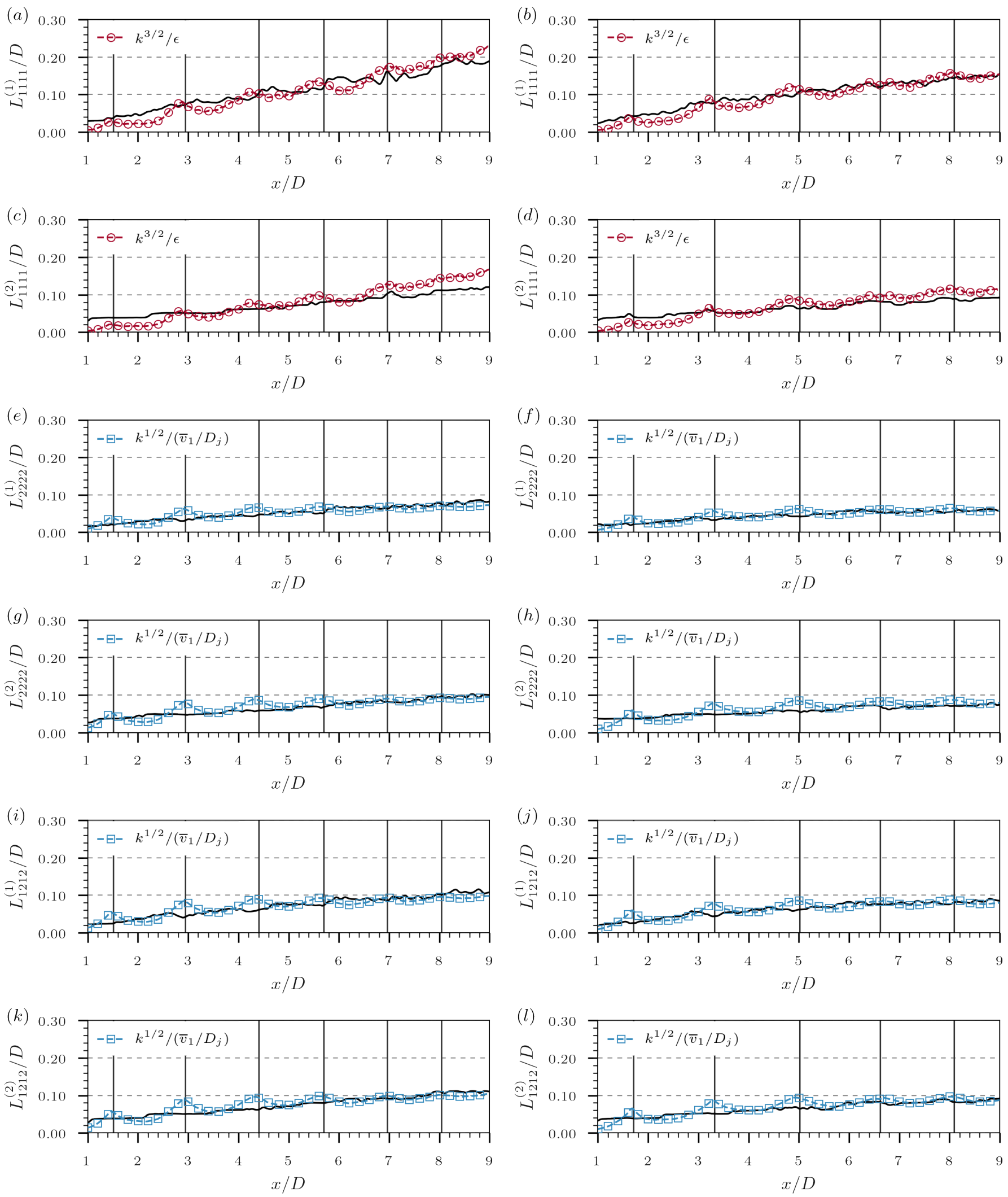

Figure 14. Distributions of the fourth-order random turbulence length scales for $M_{\mathrm{j}}$ of $(\mathbf{a}, \mathbf{c}, \mathbf{e}, \mathbf{g}) 1.45$ and $(\mathbf{b}, \mathbf{d}, \mathbf{f}, \mathbf{h}) 1.59$ 

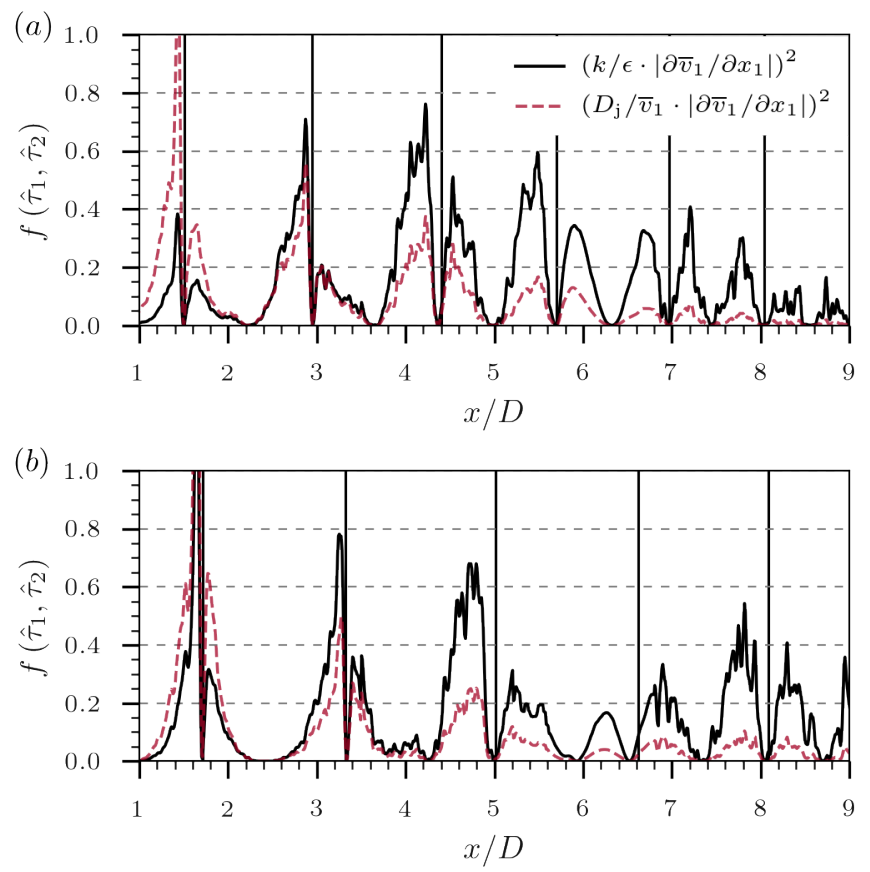

Figure 15. Streamwise distributions of the non-dimensional function $f\left(\tau_{1}, \tau_{2}\right)$ for $M_{\mathrm{j}}$ of (a) 1.45 and (b) 1.59 